\title{
Photon statistics in collective strong coupling: Nanocavities and microcavities
}

\author{
R. Sáez-Blázquez, ${ }^{1, *}$ J. Feist, ${ }^{1}$ F. J. García-Vidal,,${ }^{1,2}$ and A. I. Fernández-Domínguez ${ }^{1}$ \\ ${ }^{1}$ Departamento de Física Teórica de la Materia Condensada and Condensed Matter Physics Center (IFIMAC), \\ Universidad Autónoma de Madrid, E-28049 Madrid, Spain \\ ${ }^{2}$ Donostia International Physics Center (DIPC), E-20018 Donostia/San Sebastián, Spain
}

(Received 20 December 2017; published 25 July 2018)

\begin{abstract}
There exists a growing interest in the properties of the light generated by hybrid systems involving a mesoscopic number of emitters as a means of providing macroscopic quantum light sources. In this work, the quantum correlations of the light emitted by a collection of emitters coupled to a generic optical cavity are studied theoretically using an effective Hamiltonian approach. Starting from the single-emitter level, we analyze the persistence of photon antibunching as the ensemble size increases. Not only is the photon blockade effect identifiable, but photon antibunching originated from destructive interference processes, the so-called unconventional antibunching, is also present. We study the dependence of these two types of negative correlations on the spectral detuning between cavity and emitters, as well as its evolution as the time delay between photon detections increases. Throughout this work, the performance of plasmonic nanocavities and dielectric microcavities is compared: despite the distinct energy scales and the differences introduced by their respectively open and closed character, the bunching and antibunching phenomenology presents remarkable similarities in both types of cavities.
\end{abstract}

DOI: 10.1103/PhysRevA.98.013839

\section{INTRODUCTION}

The field of cavity quantum electrodynamics studies phenomena arising from the interaction between matter and light, the latter in the form of electromagnetic modes confined in a cavity, in the regime in which the quantum nature of the light is unveiled [1]. The matter component commonly involves twolevel systems, which describe a wide variety of emitters (from real spins to quantum dots, atoms, molecules, NV centers, or qubits, among others) as long as the state of the system can be properly represented by a two-dimensional Hilbert space. The most simple case of a single two-level system coupled to a quantized cavity mode is described by the Jaynes-Cummings model [2], later extended to an arbitrary number of emitters in the so-called Tavis-Cummings model [3]. These Hamiltonians, together with specific procedures to introduce the effect of losses [4], provide a theoretical description for a broad variety of configurations.

The interaction of light with a collection of quantum emitters has been intensively studied considering an extensive range of systems since it is of broad interest in research areas ranging from lasing $[5,6]$ and superradiance $[7,8]$ to nonclassical light generation [9], entanglement [10], or quantum information processing [11,12]. In fact, there are several optical cavity configurations capable of supporting an electromagnetic mode through semiconductor, metal, or dielectric structures, suiting thereby the specific requirements. From high-quality optical microcavities [1,13] (consisting in different planar configurations [14,15], whispering galleries [16,17], or photonic-crystal cavities [18-20], to name a few), the aim to reach higher

\footnotetext{
*rocio.saezblazquez@uam.es
}

light-matter couplings, especially on the route towards roomtemperature devices, has led to the reduction of the effective volume even below the diffraction limit of classical optics. This has given rise to nanocavities built on the basis of plasmonic nanostructures. The price to pay for the large confinement is that these structures suffer from large dissipative losses, reducing in turn the quality factor of the cavity.

The balance between coupling strength and losses leads to the well-known distinction between the weak and strong coupling regimes. Within the weak coupling regime, the principal feature consists in the enhancement of the spontaneous emission owing to the coupling of the emitters to the resonant cavity, known as the Purcell effect [21]. On the contrary, if the matter-field interaction becomes larger than the emitter and cavity relaxation rates, the system may enter the strong coupling regime, in which the genuine eigenstates turn out to be a quantum mixture of matter and light. These are referred to as dressed states or polaritons, arising due to the rapid exchange of energy between cavity and emitters. The regime of strong coupling has been demonstrated experimentally for systems involving a large amount of emitters for microcavities [22,23] as well as for plasmonic nanocavities [24,25]. Reaching this regime by involving just a single emitter becomes more challenging since it requires a cavity with a higher quality factor or stronger field confinement. While experimental realizations of single-emitter strong coupling have been reported in microcavities [26,27] and photonic crystals [28] in the past, only recently has the strong coupling regime been reached in plasmonic nanocavities with a single molecule $[29,30]$. Note that by varying the number of emitters, the system can be naturally tuned from one regime to the other; in these hybrid systems, the participation of a collection of $N$ quantum emitters makes the interaction stronger through the appearance of the 
characteristic $\sqrt{N}$ factor in the effective collective coupling strength [31].

Whereas coupling a large amount of emitters to a nanocavity mode is not difficult from the experimental perspective, addressing the complete problem theoretically presents a significant challenge. There have been some theoretical advances in the treatment of plasmonic strong coupling for large ensembles of emitters in the vanishing population regime [32]. There, the fermionic character of quantum emitters can be neglected by modeling them as bosonic harmonic oscillators and, thus, by disregarding excitonic nonlinearities and any saturation effects emerging in the photon population dynamics for the system. One step beyond this completely bosonic description was also carried out through the so-called Holstein-Primakoff approach $[33,34]$, which is equivalent to introducing a macroscopic third-order susceptibility for the medium embedding the emitter ensemble [35]. Very recently, we proposed a theoretical framework [36] able to describe plasmon-exciton strong coupling for a mesoscopic number of quantum emitters which fully accounted for their fermionic character. It is precisely the inherent quantum nonlinear character of emitters that forms the basis of many interesting phenomena when coupling light and matter, such as the well-known photon antibunching [37]. In general, intensity correlations of the emitted light are related to the probability of detecting coincident photons, so that it is used as a quantifier of multiple-photon events (the suppression of which is an important requirement for single-photon emission). Importantly, although this magnitude has been extensively used to discriminate single-photon sources, some recent papers point out that this is not by itself a reliable indicator [38].

Nonclassical light generation is extremely important in the fields of quantum cryptography [39], quantum sensing [40], quantum metrology [41], or quantum communication [42], among other emerging photonic quantum technologies. The production of single photons on demand has focused great efforts, and different methods have been used for its extraction [43], such as faint laser pulses [44], single-emitter systems [45-47], nonlinear crystals [48], or parametric downconversion [49]. All these exploit the inherent nonlinearity of the photonic system. When using a large number of emitters, their collective response becomes approximately bosonic and nonclassical light is supposed not to be generated. Nevertheless, it has been shown that when considering a mesoscopic number of emitters, these nonlinearities can be preserved and antibunched light may still be produced [50-52]. Much effort has been devoted to the analysis of the evolution of the quantum statistical properties of the light emitted by hybrid systems for increasing number of emitters, assessing the possibility of generating nonclassical light with mesoscopic ensembles. Some studies involve just a few quantum emitters [53,54], and others consider a huge amount under particular simplifications [55-57]. From a technical perspective, there exist bruteforce approaches based on Monte Carlo techniques [58,59], and developments towards the efficient treatment of large ensembles [60] thanks to the use of symmetries.

In this article, we study the coherence properties of the light radiated by a collection of $N$ identical quantum emitters placed inside a generic optical cavity when the system is coherently pumped by a laser. Two paradigmatic configurations are explored, namely, a collection of quantum dots coupled to a dielectric microcavity, and an ensemble of organic molecules within a plasmonic nanocavity (note that the pumping and the emission differ according to the open or closed character, respectively, of these systems). Whereas the former have been extensively investigated within the field of semiconductor quantum electrodynamics over the last two decades, the exploration of the latter for quantum optical purposes is still in a very early stage. To our knowledge, this article provides the first comparative study between both physical platforms for nonclassical light generation. In order to perform a meaningful comparison between both physical systems, we treat them in the same footing. In turn, this means that aspects that may be relevant in specific experimental implementations of both configurations, such as spatial and spectral inhomogeneities or emitter-emitter interactions, are neglected. The statistics of the emitted light are analyzed for these two cases determining the parameter ranges in which photon bunching and antibunching appear. In particular, the focus is on the two effects that can lead to single-photon emission: the well-known photon blockade effect and the so-called unconventional antibunching, which originates from interference effects and is thus here referred to as interference-induced correlations. On the basis of the quantum master equation for the extended Tavis-Cummings model, analytical and numerical computations are performed by using an effective Hamiltonian approach.

Our theoretical framework is described in Sec. II, beginning with the introduction of the two systems under consideration and their modeling (Sec. II A). The procedure to determine the steady state of the system (Sec. IIB) and compute the correlation functions (Sec. IIC) is described next. In Sec. III, we present a comprehensive analysis of photon statistics for realistic plasmonic nanocavities and dielectric microcavities. First, the intensity and second-order correlation function of the emitted light are explored for various ensemble sizes (Sec. III A), and analytical expressions for these magnitudes for both cavity configurations are provided. Then, the two different mechanisms leading to sub-Poissonian light are studied in detail (Sec. IIIB), followed by the analysis of the effect of spectral detuning between cavity and emitters on the photon correlations (Sec. IIIC). The evolution of the second-order correlation function at time delays different from zero is also investigated (Sec. IIID). Finally, the conclusions of the work are presented in Sec. IV.

\section{THEORETICAL FRAMEWORK}

\section{A. System}

The system under study consists of $N$ quantum emitters, modeled as simple two-level systems with a ground $\left|\mathrm{g}_{n}\right\rangle$ and an excited state $\left|e_{n}\right\rangle$, located in an optical cavity. Every emitter is coupled to a quantized single-cavity mode through the electric-dipole interaction, and they are considered not to interact among them apart from through the cavity mode (note that emitter-emitter interactions become significant only in dense ensembles [36]). A laser field coherently pumps the system, and the emitted light is collected in a detector located in the far field. An illustration of the system is depicted 

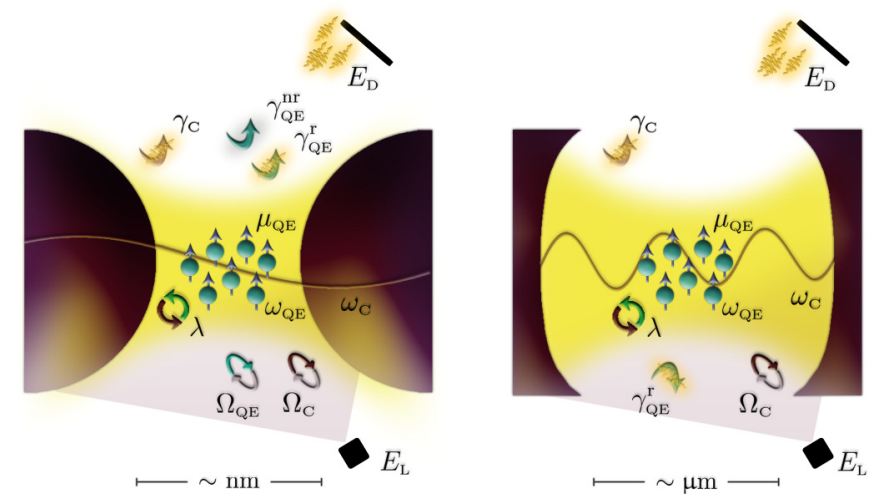

FIG. 1. Scheme of the systems, composed of a collection of quantum emitters coupled to an electromagnetic mode supported by either a nanocavity (left) or a microcavity (right). The set of parameters characterizing the system are sketched.

in Fig. 1, where the two cases of a nanocavity (left) and a microcavity (right) cavity are distinguished. Both the pumping and the emission vary according to the corresponding open or closed character: whereas for nanocavities the entire system is pumped and the radiation from both the quantum emitters and the cavity is observed (open configuration), in microcavities the coupling to the outside mode is mediated by the mirrors, such that only the cavity mode is pumped and only its emission is received at the detector (closed configuration). Apart from this fundamental distinction, the size and characteristic losses are also differentiating features between these two types of cavities. By nanocavities, we are referring to plasmonic cavities [61-64], where the spatial dimensions are reduced to the nanometer scale and cavity losses are substantial $(\sim 0.1 \mathrm{eV})$ [65]. In contrast, by microcavities we refer here to photonic crystals [20,66] and other semiconductor structures [27,67] with sizes of the order of micrometers and whose absorption is much lower $(\sim 0.1 \mathrm{meV})[13]$.

The Hamiltonian of the total system involves the excitation of both the cavity mode (with transition frequency $\omega_{\mathrm{C}}$ and bosonic creation and annihilation operators $a^{\dagger}$ and $a$ ) and the collection of $N$ quantum emitters (with transition frequency $\omega_{n}$ and creation and annihilation operators $\sigma_{n}^{\dagger}=\left|\mathrm{e}_{n}\right\rangle\left\langle\mathrm{g}_{n}\right|$ and $\sigma_{n}=\left|\mathrm{g}_{n}\right\rangle\left\langle\mathrm{e}_{n}\right|$ for the $n$th emitter which, in turn, define the operator $\sigma_{n}^{z}=\left[\sigma_{n}^{\dagger}, \sigma_{n}\right] / 2$ ), as well as the coherent pumping of a laser of frequency $\omega_{\mathrm{L}}$. In the Schrödinger picture, it reads as (from now on, $\hbar=1$ )

$$
\begin{aligned}
H= & \omega_{\mathrm{C}} a^{\dagger} a+\sum_{n=1}^{N} \omega_{n} \sigma_{n}^{z}+\sum_{n=1}^{N} \lambda_{n}\left(a^{\dagger} \sigma_{n}+a \sigma_{n}^{\dagger}\right) \\
& +\Omega_{\mathrm{C}}\left(a^{\dagger} e^{-i \omega_{\mathrm{L}} t}+a e^{i \omega_{\mathrm{L}} t}\right) \\
& +\sum_{n=1}^{N} \Omega_{n}\left(\sigma_{n}^{\dagger} e^{-i \omega_{\mathrm{L}} t}+\sigma_{n} e^{i \omega_{\mathrm{L}} t}\right),
\end{aligned}
$$

where $\lambda_{n}$ is the coupling between the $n$th quantum emitter and the cavity mode, and $\Omega_{\mathrm{C}}$ and $\Omega_{n}$ are the laser pumping to the cavity mode and the $n$th quantum emitter, respectively. If we define the effective dipole moments associated with the cavity $\boldsymbol{\mu}_{\mathrm{C}}$ and with the $n$th emitter $\boldsymbol{\mu}_{n}$, these pumping frequencies can be expressed in terms of the laser field intensity $\boldsymbol{E}_{\mathrm{L}}$ as $\Omega_{\mathrm{C}}=\boldsymbol{E}_{\mathrm{L}} \cdot \boldsymbol{\mu}_{\mathrm{C}}$ and $\Omega_{n}=\boldsymbol{E}_{\mathrm{L}} \cdot \boldsymbol{\mu}_{n}$. In the description of a microcavity, only the cavity is pumped, hence, we set $\Omega_{n}=0$ for all $n$. Although dipole-dipole interactions between emitters may become important in the nanocavity configuration when having a very dense emitter ensemble, this term will be neglected in the following either way as its inclusion does not affect significantly the conclusions drawn from our results (see discussion in Ref. [36]). Note also that the rotating wave approximation [68] has been introduced in Eq. (1), which implies that the emitter-cavity coupling is low enough to disregard the fast-rotating terms.

Through the coherent excitation, the Hamiltonian (1) acquires an explicit dependence on time, which can be easily removed by transforming it into a rotating frame. By means of the unitary operator $\mathcal{U}_{0}(t)=\exp [-i A t]$, with $A=\omega_{\mathrm{L}} a^{\dagger} a+$ $\sum_{n=1}^{N} \omega_{\mathrm{L}} \sigma_{n}^{z}$, the Hamiltonian in the transformed frame $\tilde{H}=$ $\mathcal{U}_{0}^{\dagger} H \mathcal{U}_{0}-A$ becomes

$$
\begin{aligned}
\tilde{H}= & \Delta_{\mathrm{C}} a^{\dagger} a+\sum_{n=1}^{N} \Delta_{n} \sigma_{n}^{z}+\sum_{n=1}^{N} \lambda_{n}\left(a^{\dagger} \sigma_{n}+a \sigma_{n}^{\dagger}\right) \\
& +\Omega_{\mathrm{C}}\left(a^{\dagger}+a\right)+\sum_{n=1}^{N} \Omega_{n}\left(\sigma_{n}^{\dagger}+\sigma_{n}\right),
\end{aligned}
$$

where the detunings from the laser corresponding to the cavity mode $\Delta_{\mathrm{C}}=\omega_{\mathrm{C}}-\omega_{\mathrm{L}}$ and the $n$th quantum emitter $\Delta_{n}=\omega_{n}-$ $\omega_{\mathrm{L}}$ have been defined.

When all quantum emitters have the same transition frequency $\omega_{\mathrm{QE}}$ (consequently, the same detuning $\Delta_{\mathrm{QE}}=\omega_{\mathrm{QE}}-$ $\omega_{\mathrm{L}}$ ) and equal couplings to the cavity $\lambda$ and to the laser field $\Omega_{\mathrm{QE}}=\boldsymbol{E}_{\mathrm{L}} \cdot \boldsymbol{\mu}_{\mathrm{QE}}$, the above expression for the Hamiltonian (2) reduces to

$$
\begin{aligned}
\tilde{H}= & \Delta_{\mathrm{C}} a^{\dagger} a+\Delta_{\mathrm{QE}} S^{z}+\lambda\left(a^{\dagger} S^{-}+a S^{+}\right) \\
& +\Omega_{\mathrm{C}}\left(a^{\dagger}+a\right)+\Omega_{\mathrm{QE}}\left(S^{+}+S^{-}\right),
\end{aligned}
$$

where the bright mode creation and annihilation operators $S^{+}=\sum_{n=1}^{N} \sigma_{n}^{\dagger}$ and $S^{-}=\left(S^{+}\right)^{\dagger}$, together with the operator $S^{z}=\sum_{n=1}^{N} \sigma_{n}^{z}$, have been introduced for this set of $N$ emitters. These collective operators behave like standard spin- $N / 2$ operators. Note that the coupling $\lambda$ is just the interaction between the emitter dipole moment and the near field of the cavity, that is, $\lambda=\boldsymbol{E}_{\mathrm{C}} \cdot \boldsymbol{\mu}_{\mathrm{QE}}$. Some tests beyond these assumptions, treating the emitters and their respective couplings individually, can be found in Ref. [36].

\section{B. Steady state of the system}

To study the properties of the emitted light, we first need to determine the steady state of the system. The time evolution of the density matrix $\rho$ describing the system is governed by the following master equation:

$$
\begin{aligned}
\frac{d}{d t} \rho= & -i[\tilde{H}, \rho]+\frac{\gamma_{\mathrm{C}}}{2} \mathcal{L}_{a}[\rho]+\frac{\gamma_{\mathrm{QE}}^{\mathrm{r}}}{2} \mathcal{L}_{S^{-}}[\rho] \\
& +\sum_{n=1}^{N} \frac{\gamma_{\mathrm{QE}}^{\mathrm{nr}}}{2} \mathcal{L}_{\sigma_{n}}[\rho]
\end{aligned}
$$


where the Lindblad terms, given by $\mathcal{L}_{\mathcal{O}}=2 \mathcal{O} \rho \mathcal{O}^{\dagger}-\mathcal{O}^{\dagger} \mathcal{O} \rho-$ $\rho \mathcal{O}^{\dagger} \mathcal{O}$ for an arbitrary operator $\mathcal{O}$, account for the losses arising from both cavity and quantum emitters (with decay rates $\gamma_{\mathrm{C}}$ and $\gamma_{\mathrm{QE}}$, respectively). The superscripts stand for radiative (r) and nonradiative (nr) damping. Note that while all cavity losses are included in $\mathcal{L}_{a}$, two different terms have to be added for the quantum emitters: radiative losses are described through the bright mode operator $S^{-}$(corresponding to the assumption that the emitters are at subwavelength distances and thus radiate like a collective dipole), but nonradiative losses are assigned to single emitters. Therefore, it is the single-atom operator $\sigma_{n}$ that is involved in the latter case.

In the regime of sufficiently low driving intensity, the contribution of the so-called refilling or feeding terms $\mathcal{O} \rho \mathcal{O}^{\dagger}$ appearing in the Lindblad superoperators remains negligible [36]. When they are removed, the Lindblad master equation (4) becomes equivalent to the Schrödinger equation $d|\psi\rangle / d t=$ $-i H_{\text {eff }}|\psi\rangle$ with an effective Hamiltonian

$$
H_{\mathrm{eff}}=\tilde{H}-i \frac{\gamma_{\mathrm{C}}}{2} a^{\dagger} a-i \frac{\gamma_{\mathrm{QE}}^{\mathrm{r}}}{2} S^{+} S^{-}-i \frac{\gamma_{\mathrm{QE}}^{\mathrm{nr}}}{2} S^{z},
$$

where $\tilde{H}$ is given by Eq. (3). In this effective Hamiltonian, only bright mode operators appear. Within this approach, the dark states of the ensemble, superpositions of the quantum emitter excitations that do not couple to the cavity or the external light, can thus be disregarded without further approximation. This corresponds to a crucial reduction in numerical effort when considering a large number of emitters $N$ : instead of having to consider $N$ and $N(N-1)$ states in the oneand two-excitation manifolds, respectively, only one singly and one doubly excited bright state, $\sum_{n=1}^{N} \sigma_{n}^{\dagger}|0\rangle / \sqrt{N}$ and $\sum_{n, m=1}^{N} \sigma_{n}^{\dagger} \sigma_{m}^{\dagger}|0\rangle / \sqrt{N(N-1)}$ (with $n \neq m$ ), play a role.

In the low pumping regime, we can perturbatively solve the Schrödinger equation $H_{\text {eff }}|\psi\rangle=0$ (equivalent to solving for the steady-state solution of the master equation $d \rho / d t=$ 0 ). Considering the incident laser amplitude $E_{\mathrm{L}}$ as the small parameter, the effective Hamiltonian (5) can be split as $H_{\text {eff }}=$ $H_{0}+E_{\mathrm{L}} V$, where the second term is the driving:

$$
E_{\mathrm{L}} V=\Omega_{\mathrm{C}}\left(a^{\dagger}+a\right)+\Omega_{\mathrm{QE}}\left(S^{+}+S^{-}\right) .
$$

The steady state $|\psi\rangle$ can also be expanded in a power series of $E_{\mathrm{L}},|\psi\rangle=\sum_{k=0} E_{\mathrm{L}}^{k}\left|\psi_{k}\right\rangle$. Substituting these expansions into the equation $H_{\text {eff }}|\psi\rangle=0$ and grouping terms for each power of $E_{\mathrm{L}}$ results in a set of linear equations. The zeroth-order equation leads simply to $\left|\psi_{0}\right\rangle=|0\rangle$ (that is, the ground state, which represents no excitations in the system) whereas the $k$ thorder equation turns out to be $H_{0}\left|\psi_{k}\right\rangle+V\left|\psi_{k-1}\right\rangle=0$. These equations can be successively solved so that the steady state is finally obtained from this perturbative approach.

\section{First- and second-order correlation functions}

Once the steady state is known, the correlation properties of the emitted light can be calculated. The negative-frequency part of the scattered far-field operator at the detector $\boldsymbol{E}_{\mathrm{D}}^{-}$depends on the type of cavity we consider: while for nanocavities the radiation from both cavity and quantum emitters is taken into account, $\boldsymbol{E}_{\mathrm{D}}^{-} \propto \boldsymbol{\mu}_{\mathrm{C}} a^{\dagger}+\boldsymbol{\mu}_{\mathrm{QE}} S^{+}$, for microcavities just the emission coming from the cavity is detected, $\boldsymbol{E}_{\mathrm{D}}^{-} \propto \boldsymbol{\mu}_{\mathrm{C}} a^{\dagger}$.
This reflects the open or closed character of each type of cavity. Note that the differences between the electromagnetic Green's function describing the emission from the cavity and the various emitters in nanocavities can be neglected due to their deeply subwavelength dimensions. The light intensity at a given point $I(\boldsymbol{r}, t)$ is defined in terms of the electric field operator as

$$
I(\boldsymbol{r}, t)=\left\langle\boldsymbol{E}_{\mathrm{D}}^{-}(\boldsymbol{r}, t) \boldsymbol{E}_{\mathrm{D}}^{+}(\boldsymbol{r}, t)\right\rangle
$$

and the two-time second-order correlator $G^{(2)}$ and its normalized version $g^{(2)}$ are given by [69]

$$
\begin{aligned}
& G^{(2)}\left(\boldsymbol{r}_{1}, t_{1} ; \boldsymbol{r}_{2}, t_{2}\right) \\
& \quad=\left\langle\boldsymbol{E}_{\mathrm{D}}^{-}\left(\boldsymbol{r}_{1}, t_{1}\right) \boldsymbol{E}_{\mathrm{D}}^{-}\left(\boldsymbol{r}_{2}, t_{2}\right) \boldsymbol{E}_{\mathrm{D}}^{+}\left(\boldsymbol{r}_{2}, t_{2}\right) \boldsymbol{E}_{\mathrm{D}}^{+}\left(\boldsymbol{r}_{1}, t_{1}\right)\right\rangle, \\
& g^{(2)}\left(\boldsymbol{r}_{1}, t_{1} ; \boldsymbol{r}_{2}, t_{2}\right)=\frac{G^{(2)}\left(\boldsymbol{r}_{1}, t_{1} ; \boldsymbol{r}_{2}, t_{2}\right)}{I\left(\boldsymbol{r}_{1}, t_{1}\right) I\left(\boldsymbol{r}_{2}, t_{2}\right)},
\end{aligned}
$$

where $\langle\ldots\rangle$ denotes time average. The latter is related to the (conditional) probability of detecting a photon in the detector placed at $r_{2}$ at time $t_{2}$ once a photon has reached the detector placed at $r_{1}$ at time $t_{1}$. Considering a fixed position, it can be rewritten in terms of the time delay $\tau=t_{2}-t_{1}$ as

$$
g^{(2)}(\tau)=\frac{\left\langle\boldsymbol{E}_{\mathrm{D}}^{-}(t) \boldsymbol{E}_{\mathrm{D}}^{-}(t+\tau) \boldsymbol{E}_{\mathrm{D}}^{+}(t+\tau) \boldsymbol{E}_{\mathrm{D}}^{+}(t)\right\rangle}{I(t) I(t+\tau)},
$$

which does not depend on time $t$ in the steady state. Note that for $\tau=0$, it yields the probability of detecting two coincident photons. When $g^{(2)}(\tau)<g^{(2)}(0)$, registering two photon counts with a delay $\tau$ is less likely than the observation of two simultaneous photons. This is known as photon bunching since photons tend to be distributed close together, in "bunches," instead of being located further apart. The opposite situation is given when $g^{(2)}(\tau)>g^{(2)}(0)$, known as photon antibunching, where photons tend to arrive at different times. For a coherent source of light, $g^{(2)}(\tau)=1$ for all $\tau$, which means that photons arrive independently from one another at the detector [note that $g^{(2)}(\tau) \rightarrow 1$ when $\tau \rightarrow \infty$ for any light source], leading to a Poissonian distribution of arrival times. The statistics of the light is then said to be super-Poissonian $\left[g^{(2)}(0)>1\right]$ or sub-Poissonian $\left[g^{(2)}(0)<1\right]$ if the coincidence of two photons at the detector is, respectively, more or less likely than that for a coherent light source (random case). The concepts of antibunching and sub-Poissonian statistics are often not distinguished in the literature since they usually occur together. Nevertheless, they are not equivalent concepts but reflect distinct effects; indeed, sub-Poissonian statistics can take place together with bunching [70]. Since the evolution of $g^{(2)}(\tau)$ with time delay is explored in this article, we distinguish them rigorously throughout the text to avoid misunderstandings. All the same, both antibunching and sub-Poissonian statistics are phenomena related to nonclassical light, as the conditions defining them cannot be fulfilled for classical fields [71]. Therefore, they offer a means to measure the classicality or quantumness of light.

The first- and second-order correlation functions can be evaluated by considering the perturbative solution for the steady state of the system described above. The scattering intensity $I$ and the normalized zero-delay second-order correlation function $g^{(2)}(0)$ in the steady state are thus 
computed as

$$
\begin{aligned}
I & =\left\langle\psi_{1}\left|\boldsymbol{E}_{\mathrm{D}}^{-} \boldsymbol{E}_{\mathrm{D}}^{+}\right| \psi_{1}\right\rangle, \\
g^{(2)}(0) & =\left\langle\psi_{2}\left|\boldsymbol{E}_{\mathrm{D}}^{-} \boldsymbol{E}_{\mathrm{D}}^{-} \boldsymbol{E}_{\mathrm{D}}^{+} \boldsymbol{E}_{\mathrm{D}}^{+}\right| \psi_{2}\right\rangle / I^{2} .
\end{aligned}
$$

From these equations, it follows that our perturbative calculations can be restricted to second order, and we can truncate the Hilbert space at the two-excitation manifold. To compute the second-order correlation function $g^{(2)}(\tau)$, the evolution operator $\mathcal{U}(t)=\exp \left[-i H_{\mathrm{eff}} t\right]$ has to be introduced:

$$
\begin{aligned}
g^{(2)}(\tau) & =\left\langle\boldsymbol{E}_{\mathrm{D}}^{-}(0) \boldsymbol{E}_{\mathrm{D}}^{-}(\tau) \boldsymbol{E}_{\mathrm{D}}^{+}(\tau) \boldsymbol{E}_{\mathrm{D}}^{+}(0)\right\rangle / I^{2} \\
& =\left\langle\psi\left|\boldsymbol{E}_{\mathrm{D}}^{-} \mathcal{U}^{\dagger}(\tau) \boldsymbol{E}_{\mathrm{D}}^{-} \boldsymbol{E}_{\mathrm{D}}^{+} \mathcal{U}(\tau) \boldsymbol{E}_{\mathrm{D}}^{+}\right| \psi\right\rangle / I^{2},
\end{aligned}
$$

where $\boldsymbol{E}_{\mathrm{D}}^{-} \equiv \boldsymbol{E}_{\mathrm{D}}^{-}(0)$, and the perturbative solution of $|\psi\rangle$ up to second order is also used in the calculation.

\section{RESULTS AND DISCUSSION}

In the following, the theoretical framework presented in the previous section is applied to study the coherence properties of the light emitted by a collection of $N$ quantum emitters coupled to either a nanocavity or a microcavity, with the distinction introduced before. Our attention is focused on resonant coupling, setting $\omega_{\mathrm{C}}, \omega_{\mathrm{QE}} \equiv \omega_{0}=3 \mathrm{eV}$ in all cases (except in the section where the effects of spectral detuning are explored). Beyond that, we have to consider a specific set of parameters for each system. The dissipation rate associated with the microcavity is taken to be $\gamma_{\mathrm{C}}=66 \mu \mathrm{eV}$ (16 GHz) [9], which corresponds to the spontaneous decay rate of a dipole moment $\mu_{\mathrm{C}}=$ $3.1 \mathrm{e} \mathrm{nm} \mathrm{[72].} \mathrm{On} \mathrm{the} \mathrm{contrary,} \mathrm{substantial} \mathrm{nonradiative} \mathrm{losses}$ are a distinctive feature of plasmonic cavities $[61,73]$, hence, we set $\gamma_{\mathrm{C}}=0.1 \mathrm{eV}(24 \mathrm{THz})$ for the nanocavity. This value also incorporates the radiative losses corresponding to a dipole with $\mu_{\mathrm{C}}=19 \mathrm{e} \mathrm{nm}$, which mimics the cavity emission [74]. Regarding the emitters, we consider those typically used in the experimental setups involving each cavity. First, the quantum emitters usually located inside dielectric cavities are characterized by negligible nonradiative losses, thus, $\gamma_{\mathrm{QE}}^{\mathrm{nr}}=0$. In particular, we choose semiconductor quantum dots with dipole moment $\mu_{\mathrm{QE}}=0.25$ e nm, which corresponds to a radiative decay rate $\gamma_{\mathrm{OE}}^{\mathrm{r}}=0.41 \mu \mathrm{eV}(0.10 \mathrm{GHz})$ [9]. Conversely, the quantum emitters interacting with plasmonic nanocavities are considered to be organic molecules with $\mu_{\mathrm{QE}}=1 \mathrm{e} \mathrm{nm}$, and presenting very low quantum yield. The specific rates chosen for these emitters are $\gamma_{\mathrm{QE}}^{\mathrm{nr}}=15 \mathrm{meV}(3.6 \mathrm{THz})$ and $\gamma_{\mathrm{QE}}^{\mathrm{r}}=$ $6 \mu \mathrm{eV}(1.5 \mathrm{GHz})$. Note that a rather large nonradiative decay rate was considered in order to account for the quantum-yield reduction experienced by organic molecules arranged in dense ensembles [25]. A more extended study on the QE parameters for the nanocavity case can be found in [36].

In our study, we assume that both open and closed cavities operate at low temperature, which diminishes greatly the impact of pure-dephasing processes in the dynamics of both quantum dots [75] and organic molecules [76]. Accordingly, we have not included this decoherence mechanism in our theoretical model. Moreover, from here on and for simplicity, we consider that the external laser field $\boldsymbol{E}_{\mathrm{L}}$ is parallel to both the cavity and the quantum emitter dipole moments. Note that this turns out to be the optimal configuration to enter the strong coupling regime.

\section{A. Intensity and coherence}

The features of the light emerging from these two configurations are studied first as a function of the number of emitters. The intensity and the zero-delay second-order correlation function for the steady state are computed from Eq. (10). Although the numerical results displayed below depend on the particular values of the parameters, the qualitative picture we present is not bound to the specific configuration; on the contrary, it remains the same when considering a wide range of parameters describing realistic systems.

\section{Plasmonic nanocavities}

We consider first the nanocavity, where the light reaching the detector comes from both the quantum emitters and the cavity itself. Fig. 2 shows the scattering intensity $I$ (top row) and the zero-delay second-order correlation function $g^{(2)}(0)$ (bottom row) for three different collections of emitters: $N=1$ (a), 5 (b), and 25 (c). Both magnitudes are plotted as a function of the laser detuning $\omega_{\mathrm{L}}-\omega_{0}$ and the coupling strength $\lambda$, which is expressed in units of the cavity decay rate $\gamma_{\mathrm{C}}$.

In all intensity maps, two scattering maxima are observed, which correspond to the polariton energies within the strong coupling regime, that is, the eigenenergies of the dressed states in the one-excitation manifold, the first rung of the so-called Tavis-Cummings ladder. In this way, for each value of the coupling strength we find two intensity peaks at laser frequencies that match the lower (LP) and the upper (UP) polariton energies. These dispersion curves are plotted in dotted lines overlapping the maps, so that the correspondence is easily observed. Note that these two maxima branches are also apparent within the weak coupling regime, and thus this presence cannot be regarded as an energy splitting. Its origin actually lies in a Fano-like interference, appearing when two signals with very different linewidths interact [77-79]. The pronounced minimum in the scattering intensity is in this case produced by the destructive interference between the cavity and emitter emission.

Finally, observe the asymmetry between the two intensity maxima branches: the one corresponding to the UP is distinctly brighter. Note that the emission coming from each polariton can be described from either the parallel (UP) or antiparallel (LP) superposition of the dipole moments associated with the plasmon and the bright mode of the emitter ensemble. Since the dipole moment corresponding to the UP is larger, its emission is more intense. This difference in the effective dipole moment between LP and UP grows as $N$ increases, as a consequence of the greater collective dipole moment of the ensemble. Hence, the contrast between branches becomes more pronounced for larger ensemble sizes.

We can get a better understanding from the analytical results obtained thanks to the perturbative approach described above. The expression for the scattered intensity reads as

$$
I \propto\left|\frac{\tilde{\Delta}_{\mathrm{C}} \mu_{\mathrm{QE}}^{2}+\tilde{\Delta}_{\mathrm{QE}} \mu_{\mathrm{C}}^{2} / N-2 \lambda \mu_{\mathrm{C}} \mu_{\mathrm{QE}}}{\tilde{\Delta}_{\mathrm{C}} \tilde{\Delta}_{\mathrm{QE}} / N-\lambda^{2}}\right|^{2},
$$

where the detunings of the laser frequency from both the cavity $\Delta_{\mathrm{C}}$ and the emitters $\Delta_{\mathrm{QE}}$ are redefined to introduce the associated losses as $\tilde{\Delta}_{\mathrm{C}}=\Delta_{\mathrm{C}}-i \gamma_{\mathrm{C}} / 2$ and 


$$
\mathrm{N}=1
$$
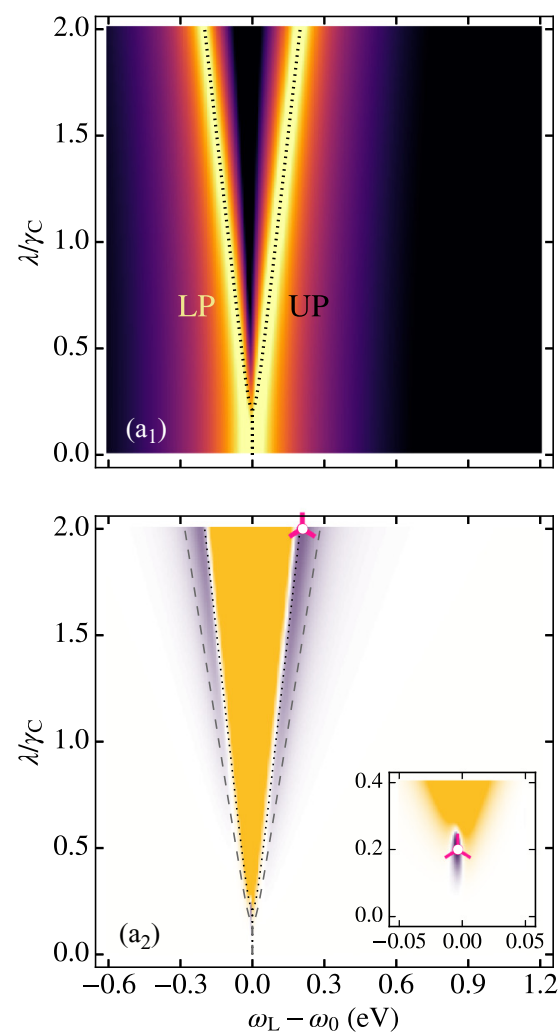

$\mathrm{N}=5$
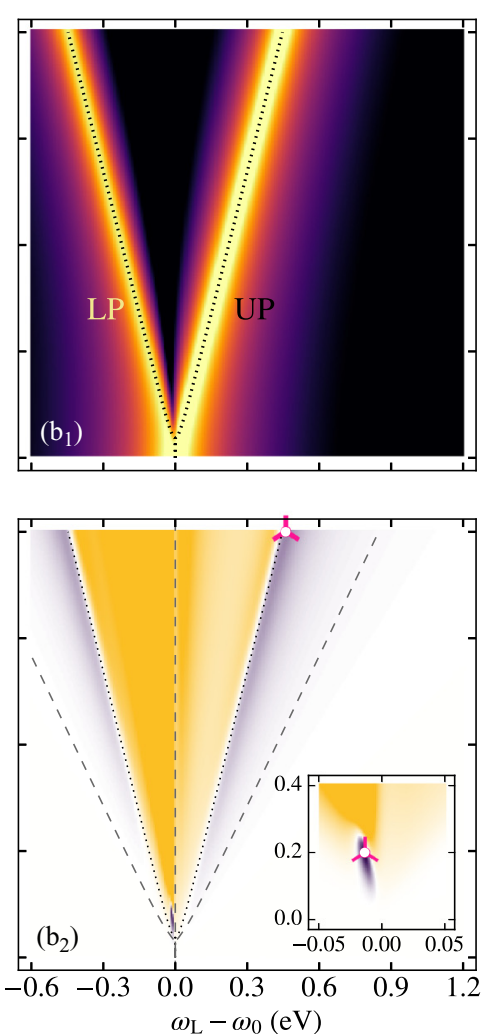

$\mathrm{N}=25$
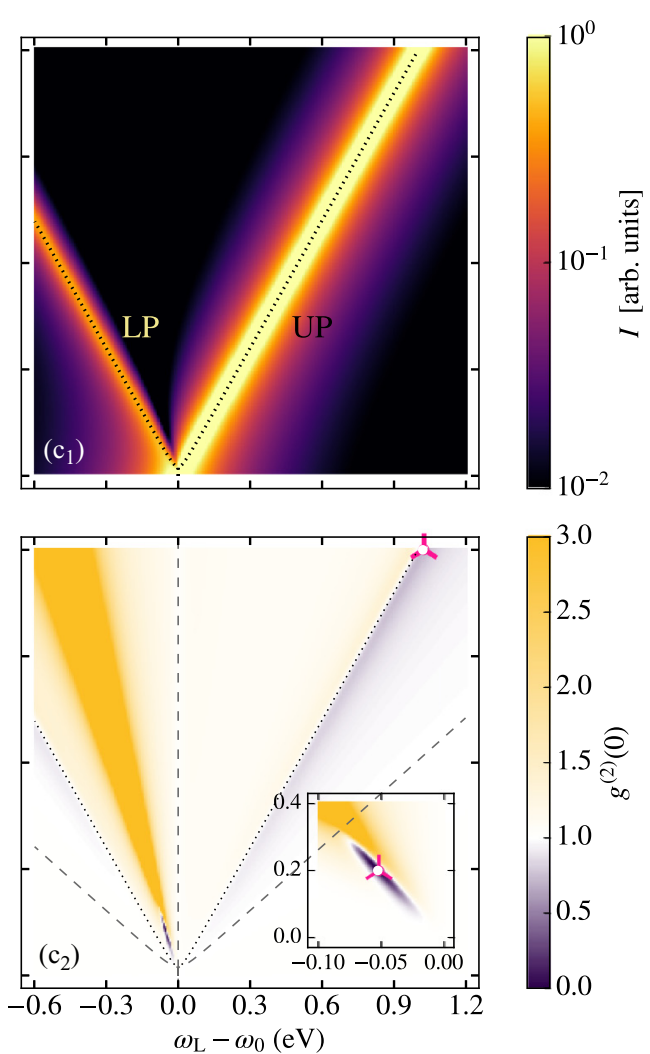

FIG. 2. Scattering intensity $I$ (top row) and correlation function $g^{(2)}(0)$ (bottom row) versus laser detuning $\omega_{\mathrm{L}}-\omega_{0}$ and coupling strength $\lambda$ (in units of the cavity decay rate $\gamma_{\mathrm{C}}$ ) for a system of $N=1$ (a), 5 (b), and 25 (c) quantum emitters coupled to a plasmonic nanocavity. In these panels, dotted (dashed) lines plot the polariton frequencies (half-frequencies) in the one-excitation (two-excitation) manifold. Insets zoom into the low coupling region. Magenta marks indicate points whose $g^{(2)}(\tau)$ is plotted in Fig. $8\left(\mathrm{a}_{1}\right)$.

$\tilde{\Delta}_{\mathrm{QE}}=\Delta_{\mathrm{QE}}-i\left(\gamma_{\mathrm{OE}}^{\mathrm{nr}}+N \gamma_{\mathrm{QE}}^{\mathrm{r}}\right) / 2$. This expression confirms the origin of the intensity maxima: the condition for which the denominator vanishes, $\lambda^{2}=\tilde{\Delta}_{\mathrm{C}} \tilde{\Delta}_{\mathrm{QE}} / N$, gives us the dispersion of the LP and UP. Notice the $\sqrt{N}$ dependence, characteristic scaling of collective coupling. In addition, this expression sheds light into the asymmetry in the branches: the intensity behaves as $I \propto\left(1 \mp \sqrt{N} \mu_{\mathrm{QE}} / \mu_{\mathrm{C}}\right)^{2}$ for the $\mathrm{LP}$ (upper sign) and UP (lower sign) when the losses from both cavity and emitters are neglected.

The bottom row of Fig. 2 clearly shows areas of superPoissonian (yellow colored) as well as sub-Poissonian (blue colored) statistics for all ensembles sizes. Focusing our attention first in the single-emitter case $(N=1)$, we distinguish a main super-Poissonian area located between the LP and UP energies (again depicted as dotted lines) for all coupling values. Close to these polariton frequencies, but still far from the twoexcitation eigenenergies (depicted as dashed lines), regions of sub-Poissonian light are found, being more pronounced as the coupling strengthens. These correspond to the well-known photon blockade effect, where the presence of an excitation in the system prevents the absorption of a second photon at certain frequencies due to the anharmonicity of the energy ladder. Apart from these three stripes, we find another area of subPoissonian emission that is enlarged in the corresponding inset. It lies around the resonant frequency $\omega_{\mathrm{L}}=\omega_{0}$. The mechanism behind it was addressed theoretically in the context of dielectric microcavities [80], the so-called unconventional antibunching. Here, we employ the term interference-induced correlations since it is the destructive interference among possible decay paths that produces the suppression of two-photon processes and hence the drop of $g^{(2)}(0)$ below one $[81,82]$. In the following section, these two different types of sub-Poissonian light are discussed in further detail.

The statistical features observed for single emitters are also present for larger $N$. As we already pointed out in Ref. [36], photon correlations arising at the single-emitter level remain, and can even be enhanced as the ensemble size increases. This is observed in the panels corresponding to $N=5$ and 25 in Fig. 2. The area of bunched light remains between the one-excitation eigenenergies, although it tends to approach the LP branch when the number of emitters increases. This tilt is also observed for the interference-induced correlation area: the region of negative correlations shifts towards the LP energy, while values for the function $g^{(2)}(0)$ below one are still achieved within the same coupling range as for the single-emitter case. Note that by increasing further the number of emitters, the system eventually bosonizes [that is, it yields $\left.g^{(2)}(0)=1\right]$. The quantum character of the emitted light is then lost. Focusing now on the region associated with the photon blockade effect, we observe that the minimum following the 
(a) Open configuration
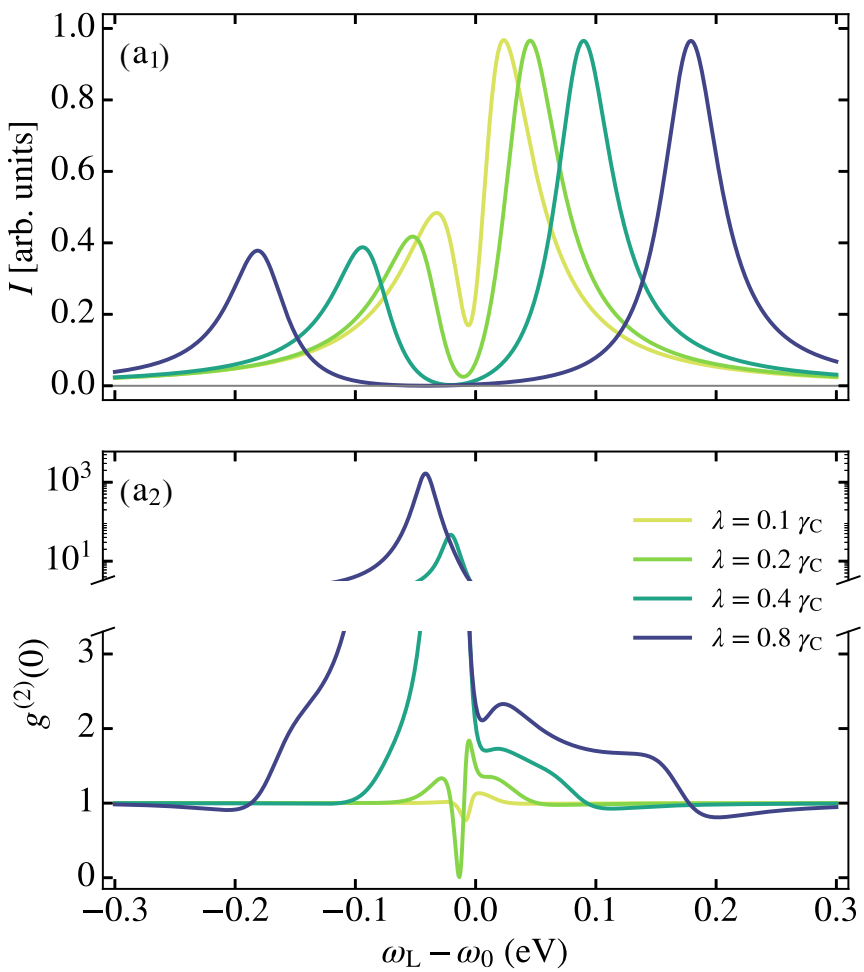

(b) Closed configuration
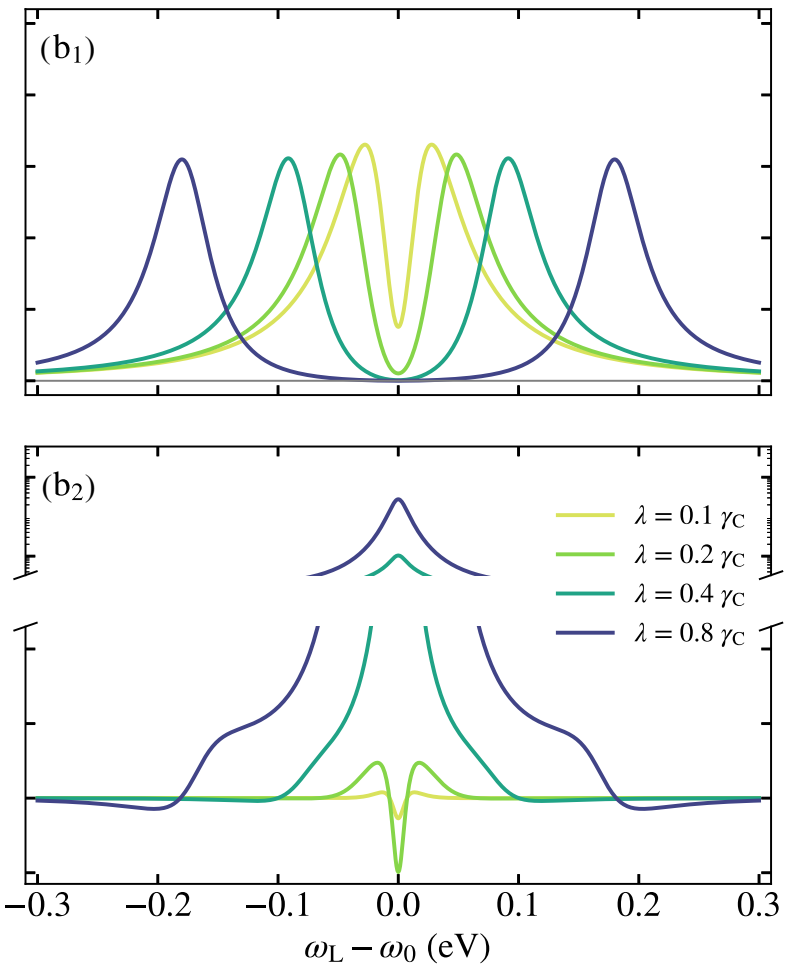

FIG. 3. Scattering intensity $I$ (top row) and correlation function $g^{(2)}(0)$ (bottom row) versus laser detuning $\omega_{\mathrm{L}}-\omega_{0}$ for a system of $N=5$ quantum emitters coupled to a plasmonic nanocavity for various coupling strengths $\lambda$ with (a) and without (b) considering the pumping to and the emission from the quantum emitters.

UP branch is deeper than the LP one. Nevertheless, it is apparent how both fade for larger sizes of the emitter ensemble. Indeed, for $N=25$ there are a wide range of coupling strengths where this effect is not observable. On the contrary, for moderate values of the coupling, the dip corresponding to interference effects is not only present, but becomes quite pronounced.

From our perturbative approach, we can also obtain the analytical expression for the correlation function $g^{(2)}(0)$ corresponding to an open nanocavity:

$g^{(2)}(0)=\mid 1-\frac{1}{N}\left(\frac{\tilde{\Delta}_{\mathrm{C}} \mu_{\mathrm{QE}}-\lambda \mu_{\mathrm{C}}}{\tilde{\Delta}_{\mathrm{C}} \mu_{\mathrm{QE}}^{2}+\tilde{\Delta}_{\mathrm{QE}} \mu_{\mathrm{C}}^{2} / N-2 \lambda \mu_{\mathrm{C}} \mu_{\mathrm{QE}}}\right)^{2} \frac{\left(\tilde{\Delta}_{\mathrm{QE}}+i N \gamma_{\mathrm{QE}}^{\mathrm{r}} / 2\right)\left[\tilde{\Delta}_{\mathrm{C}} \tilde{\Delta}_{\mathrm{QE}} \mu_{\mathrm{QE}}^{2}+\left(\tilde{\Delta}_{\mathrm{C}} \mu_{\mathrm{QE}}-\lambda \mu_{\mathrm{C}}\right)^{2}-N \lambda^{2} \mu_{\mathrm{QE}}^{2}\right]}{\left(\tilde{\Delta}_{\mathrm{QE}}+i \gamma_{\mathrm{QE}}^{\mathrm{r}} / 2\right)\left(\tilde{\Delta}_{\mathrm{C}}^{2}+\tilde{\Delta}_{\mathrm{C}} \tilde{\Delta}_{\mathrm{QE}}-N \lambda^{2}\right)-\tilde{\Delta}_{\mathrm{C}}(N-1) \lambda^{2}}$

where, again, we have made use of the redefined detunings $\tilde{\Delta}_{\mathrm{C}}$ and $\tilde{\Delta}_{\mathrm{QE}}$. We observe that, as expected, $g^{(2)}(0) \rightarrow 1$ when $N$ tends to infinity for a fixed value of the coupling strength, so the expression does recover the bosonization limit. Notice also that the denominator of the first term coincides with the numerator of the analytical expression for the intensity [Eq. (12)], and the vanishing condition for the denominator of the second term yields the polaritons of the second rung of the Tavis-Cummings ladder.

Considering the same cavity and emitters as in the plasmonic case, we can explore the changes introduced when quantum emitters are not directly pumped by the laser and only the radiation coming from the cavity is registered at the detector. This mimics the setup of a closed microcavity for the parameter values distinctive of a plasmonic nanocavity.
Results for the intensity $I$ and the correlation function $g^{(2)}(0)$ for different values of the coupling strength $\lambda$ are shown in Fig. 3 for these two situations: with (a) and without (b) considering both the pumping and the emission associated with the quantum emitters. To make the comparison, we present the particular case of a collection of $N=5$ quantum emitters. Therefore, the lines appearing in the left-hand column of Fig. 3 are just cuts of the maps $\left(b_{1}\right)$ and $\left(b_{2}\right)$ of Fig. 2 at four particular values of the coupling strength.

First, we notice that the asymmetry in the two intensity peaks is removed when considering only the emission from the cavity. As commented before, for an open nanocavity, the effective dipole moment of the LP and the UP are, respectively, the parallel and antiparallel superpositions of those of the cavity and the quantum emitters. This makes the emission of 

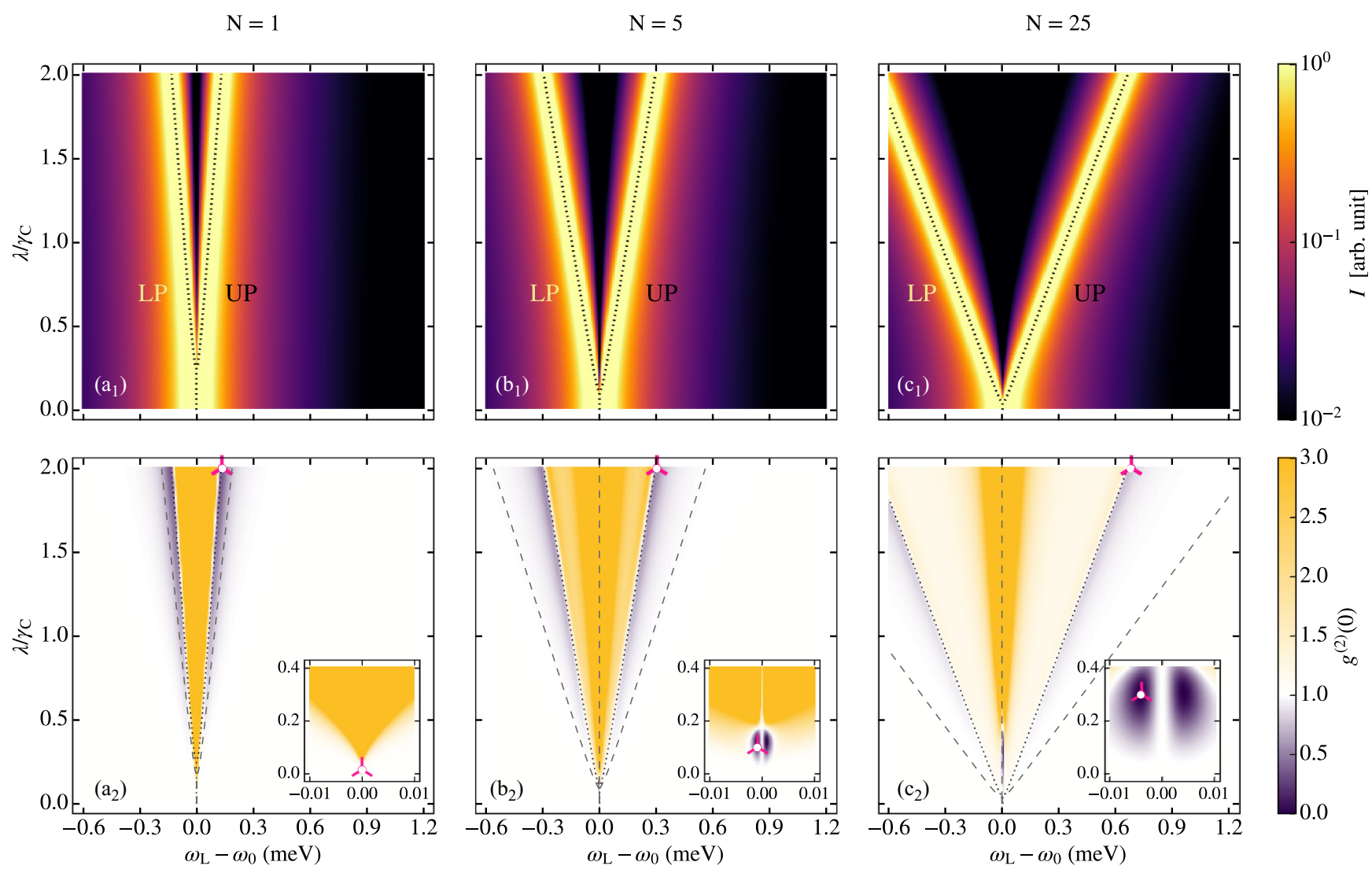

FIG. 4. Scattering intensity $I$ (top row) and correlation function $g^{(2)}(0)$ (bottom row) versus laser detuning $\omega_{\mathrm{L}}-\omega_{0}$ and coupling strength $\lambda$ (in units of the cavity decay rate $\gamma_{\mathrm{C}}$ ) for a system of $N=1$ (a), 5 (b), and 25 (c) quantum emitters coupled to a dielectric microcavity. In these panels, dotted (dashed) lines plot the polariton frequencies (half-frequencies) in the one-excitation (two-excitation) manifold. Insets zoom into the low coupling region. Magenta marks indicate points whose $g^{(2)}(\tau)$ is plotted in Fig. $8\left(b_{1}\right)$.

the UP brighter, and it also introduces a noticeable dependence on the number of emitters. On the contrary, in this new configuration both polaritons radiate with the same associated dipole moment (corresponding to the cavity, which is the same regardless the ensemble size), and the associated emission is thus identical. Notice also that the positions of the intensity peaks are the same for open and closed configurations because the polariton energies do not change.

The symmetry observed in the intensity patterns is also kept in the correlation function $g^{(2)}(0)$. Apart from this difference, the main features in the statistics are kept from the open case, namely, first, around the zero value of the laser detuning $\left(\omega_{\mathrm{L}}=\omega_{0}\right)$ there exists a dip for reduced coupling strength whereas a maximum is developed as the interaction increases, and second, for frequencies near the one-excitation polaritons, the photon blockade effect is observable. From these cuts in Fig. 3( $\left.\mathrm{a}_{2}\right)$, we confirm that the minimum following the UP branch is the deepest.

Finally, note that there are two important features characterizing the QEs in the nanocavity case that can affect the results reported above: the nonradiative decay associated with organic molecules in dense arrangements and the growing emitter-emitter interactions due to the limited space in which the ensemble is placed. It was already shown in [36] that antibunching areas are favored by large emitter losses, thus, the variation of this parameter would have an effect on the size of the parametric area where strong negative photon correlations occur. On the contrary, antibunching was shown to be quite robust against dipole-dipole interactions between emitters, and only when interaction becomes considerably large may the degree of antibunching be slightly reduced.

\section{Dielectric microcavities}

Now, we consider the typical configuration of dielectric microcavities, where only the cavity mode is pumped and direct emission from the quantum emitters does not take place (closed configuration). A study similar to the previous section is carried out, determing the intensity $I$ and the zero-delay second-order correlation function $g^{(2)}(0)$. The results are shown in Fig. 4 for the same three cases: $N=1$ (a), 5 (b), and 25 (c) quantum emitters placed inside the cavity.

The intensity panels reveal again the presence of the two polaritons when entering the strong coupling regime. The energies corresponding to the dressed states in the one-excitation manifold are plotted in dotted lines, and they overlap the intensity peaks. Nevertheless, in contrast to the nanocavity configuration, these two intensity maxima are symmetric and barely vary their height as the number of emitters increases. As we have commented in the previous section, this is due to the fact that only the emission from the cavity is registered, so the effective radiating dipole moment is always the same. 
This underlying symmetry is also revealed in the analytical expression of the intensity, computed from the perturbative approach

$$
I \propto\left|\frac{\tilde{\Delta}_{\mathrm{QE}} \mu_{\mathrm{C}}^{2} / N}{\tilde{\Delta}_{\mathrm{C}} \tilde{\Delta}_{\mathrm{QE}} / N-\lambda^{2}}\right|^{2}
$$

This expression can be reproduced from that corresponding to nanocavities [Eq. (12)] just by considering the limit $\mu_{\mathrm{QE}} \rightarrow 0$. We observe that the denominator remains unchanged, so its vanishing condition gives us again the energy dispersion for the polaritons and hence the position of the intensity maxima.

The results for the correlation function $g^{(2)}(0)$ depicted in the bottom row of Fig. 4 show a similar pattern to the ones found for nanocavities (Fig. 2), although incorporating the symmetry already expected. Around the zero laser detuning $\left(\omega_{\mathrm{L}}=\omega_{0}\right)$ and for intermediate (or large) coupling strengths we find super-Poissonian statistics (yellow colored). These panels also show the two types of sub-Poissonian emission (blue colored) previously observed: that associated with the phenomenon of photon blockade, as well as that related to destructive interference. The positions of the eigenenergies corresponding both to the one- and two-excitation manifolds are plotted in dotted and dashed lines, respectively, overlapping the correlation maps. The frequencies where the photon blockade effect occurs are easily relatable next to the dotted lines. Nevertheless, it is in the other area of sub-Poissonian emission, the one associated with interference effects, where the main difference between open and closed cavities appears. Apart from the spectral symmetry already discussed, the development of two dips instead of a single one is the most apparent feature. For lower values of the coupling strength, we find $g^{(2)}(0)=1$ at zero detuning, while on both sides of this frequency, a window with sub-Poissonian emission is visible. The presence of this double-dip pattern disappears when introducing nonradiative losses associated with the quantum emitters.

The evolution of correlations as the number of emitters increases differs from the open nanocavity case. Apart from the fact that symmetry modifies the laser frequencies at which the different regions are achieved (for a specific value of the coupling strength), the main variation concerns the subPoissonian emission caused by destructive interference. These areas are enlarged in the insets of Fig. 4. As the ensemble size increases, the parameter ranges in which we find $g^{(2)}(0)<1$ clearly widen. Therefore, it is possible to obtain antibunched emission for a specific coupling strength just by increasing the number of emitters. Beyond a particular $N$, the system tends to reach the bosonization limit, where $g^{(2)}(0)=1$. The onset of this regime depends on the coupling strength between cavity and emitters and, as seen in Fig. 4( $\left.\mathrm{c}_{2}\right)$, for $N=25$ emitters we still find significant negative correlations for a wide interval of coupling values. Note that the photon blockade region does not endure so long and it practically disappears for a few emitters within this coupling range.

There exists a major aspect, not mentioned before, that should be highlighted: the range of laser detunings at which this nonclassical behavior is found is of the order of meV. Notice that the energy scale in Fig. 4 differs in three orders of magnitude from the one corresponding to nanocavities, Fig. 2. Therefore, the spectral robustness and accessibility of the antibunched regions is significantly different in open and closed cavities, especially in the case of interferenceinduced negative correlations. We anticipate that the spectrally broad (narrow) nature of photon correlations in plasmonic nanocavities (dielectric microcavities) implies a faster (slower) temporal evolution of $g^{(2)}(\tau)$.

Finally, to gain insight into the coherence properties discussed above, we present the analytical expression for the correlation function $g^{(2)}(0)$ :

$$
g^{(2)}(0)=\left|1-\frac{1}{N}\left(\frac{\lambda}{\tilde{\Delta}_{\mathrm{QE}} / N}\right)^{2} \frac{\left(\tilde{\Delta}_{\mathrm{QE}}+i N \gamma_{\mathrm{QE}}^{\mathrm{r}} / 2\right) \lambda^{2}}{\left(\tilde{\Delta}_{\mathrm{QE}}+i \gamma_{\mathrm{QE}}^{\mathrm{r}} / 2\right)\left(\tilde{\Delta}_{\mathrm{C}}^{2}+\tilde{\Delta}_{\mathrm{C}} \tilde{\Delta}_{\mathrm{QE}}-N \lambda^{2}\right)-\lambda^{2} \tilde{\Delta}_{\mathrm{C}}(N-1)}\right|^{2},
$$

which can be also obtained by taking $\mu_{\mathrm{QE}} \rightarrow 0$ in Eq. (13). Again, this expression yields $g^{(2)}(0)=1$ when $N \rightarrow \infty$, so the classical behavior is recovered in this limit. Note as well that we find $g^{(2)}(0)=1$ at the resonant frequency $\omega_{\mathrm{L}}=\omega_{0}$ when all losses are neglected.

\section{B. Two different mechanisms leading to sub-Poissonian light}

Studying photon correlations in coupled systems, we have identified two types of sub-Poissonian emission appearing in both nanocavities and microcavities. In order to shed light into their different nature, we proceed in this section to examine their emergence in more detail. In Fig. 5, the focus is on the photon blockade effect, which takes place close to the polarition energies of the one-excitation manifold, whereas in Fig. 6 we study the sub-Poissonian emission associated with destructive interference, which appears for moderate coupling strength in the region of zero detuning. The population, the intensity $I$, and the correlation functions $G^{(2)}(0)$ and $g^{(2)}(0)$ are plotted as a function of the laser detuning $\omega_{\mathrm{L}}-\omega_{0}$ for nanocavities (left-hand-side panels) and microcavities (righthand-side panels) at specific coupling strengths to explore these processes.

\section{Photon blockade}

The sub-Poissonian emission due to the photon blockade effect originates from the anharmonicity of the TavisCummings ladder, as we have commented before. When the laser has an energy close to that of one of the polaritons at the one-excitation manifold, the population of this particular hybrid state increases. In Figs. 5( $\left.a_{1}\right)$ and 5( $\left.b_{1}\right)$, populations are plotted in the basis of the dressed states. There, the continuous colored lines, corresponding to the population of the UP (dark pink) and the LP (light pink), experience an increase when the laser frequency is tuned to be in the vicinity of the 
(a) Plasmonic nanocavities
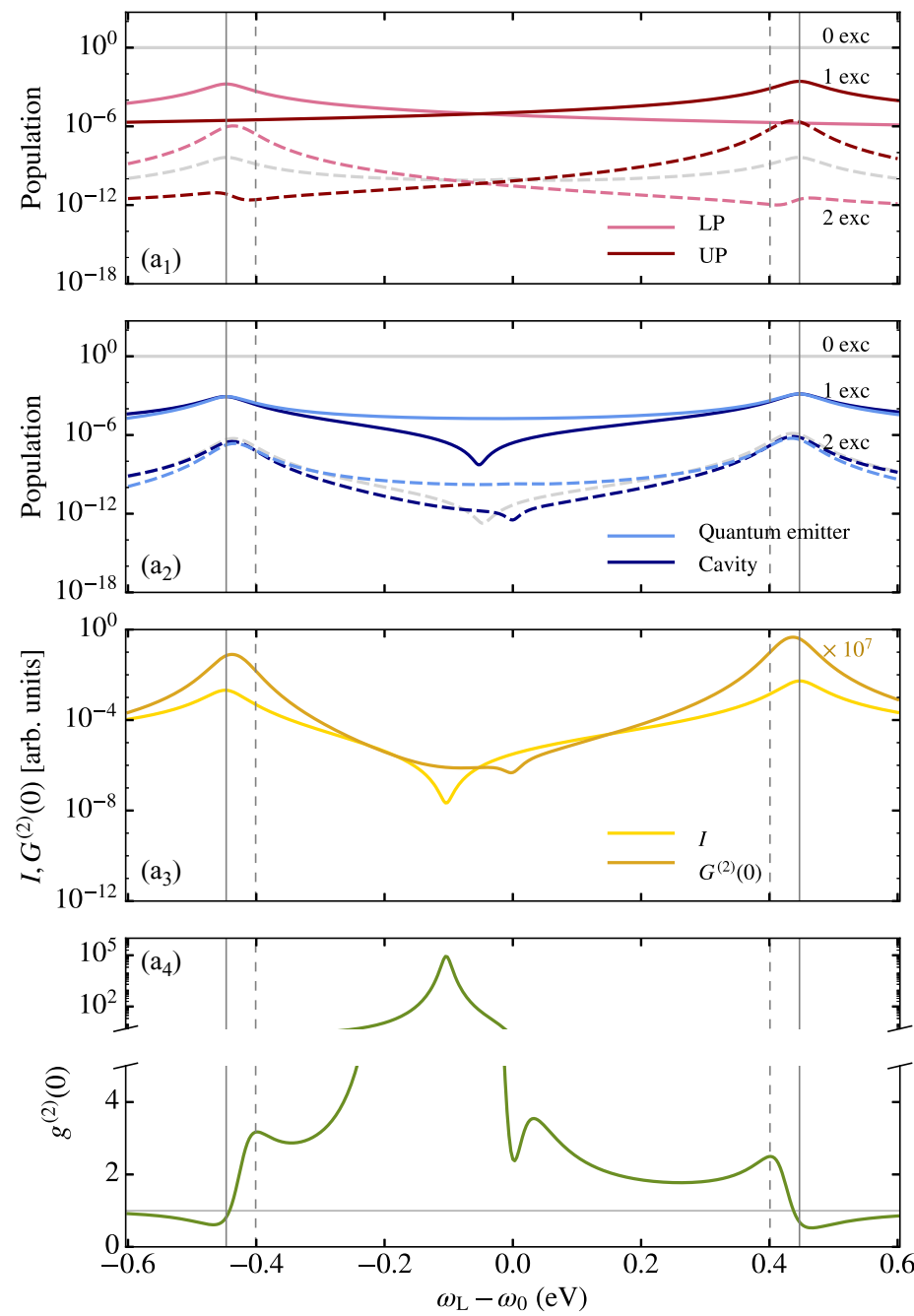

(b) Semiconductor microcavities
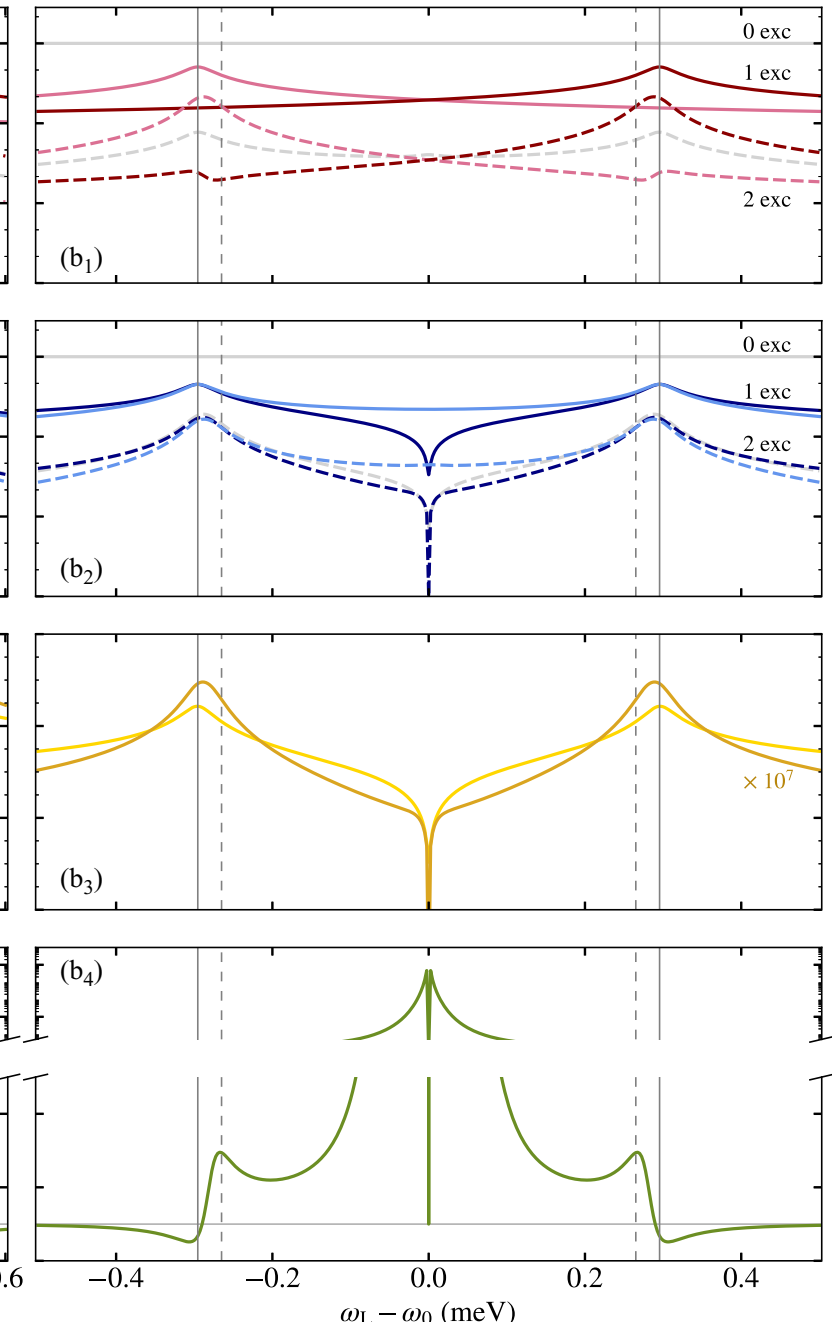

FIG. 5. Population [in the polariton basis (first row) and in the cavity-emitters basis (second row)], intensity $I$, and correlation functions $G^{(2)}(0)$ and $g^{(2)}(0)$ versus laser detuning $\omega_{\mathrm{L}}-\omega_{0}$ for a system of $N=5$ quantum emitters coupled to a nanocavity (a) and a microcavity (b) at coupling strength $\lambda / \gamma_{\mathrm{C}}=2$ (for which the photon blockade effect appears). In these panels, continuous vertical gray lines indicate the polariton frequencies in the one-excitation manifold, while the dashed ones represent the energy differences between the state of one LP and one UP (belonging to the two-excitation manifold) and the state with either one LP (left dashed line) or one UP (right dashed line).

corresponding polariton frequency (continuous vertical gray lines). Nevertheless, for these specific energies, the laser is out of resonance for promoting the state from the one- to the twoexcitation manifold (dashed vertical gray lines depict these energy differences). This diminishes the probability of emission of two simultaneous photons, leading to sub-Poissonian statistics. These panels also show, in dashed colored lines, the populations of the states belonging to the two-excitation manifold: two LPs (light pink), one LP and one UP (very light gray), and two UPs (dark pink). Note that the maxima of these curves are not located exactly at the polariton frequencies. They are slightly shifted as a consequence of the energy differences between the one- and the two-excitation manifolds.

When the populations are expressed in terms of the cavity and emitter states, Figs. 5( $\left.\mathrm{a}_{2}\right)$ and 5( $\left.\mathrm{b}_{2}\right)$, all curves belonging to the same subspace (continuous or dashed lines for the one- and two-excitation manifolds, respectively) seem to converge to the same value at the frequencies where the photon blockade phe- nomenon takes place (that is, near the polariton frequencies). Apart from that, we observe that there exist two clear minima in the population curves corresponding to the state with one (continuous dark blue line) and two (dashed dark blue line) excitations in the cavity. Each of them has a replica in one of the curves depicted in Figs. $5\left(\mathrm{a}_{3}\right)$ and $5\left(\mathrm{~b}_{3}\right)$. This is especially visible for the nanocavities where these two minima do not coincide. Indeed, the intensity (yellow line) and the $G^{(2)}(0)$ (ocher line) functions reproduce the form of the populations of the states with one and two excitations in the cavity mode, respectively. The origin of this correspondence is clear for the closed configuration (as only the emission from the cavity is detected). For the open one, it results from the fact that the dipole moment of the cavity is greater than the collective dipole of the emitter ensemble. Thus, the former contributes the most to the emitted light (for a reduced number of emitters). Note that intensity accounts for one-photon processes, while $G^{(2)}(0)$ reflects from two-photon processes instead [Eq. (10)]. 
(a) Plasmonic nanocavities
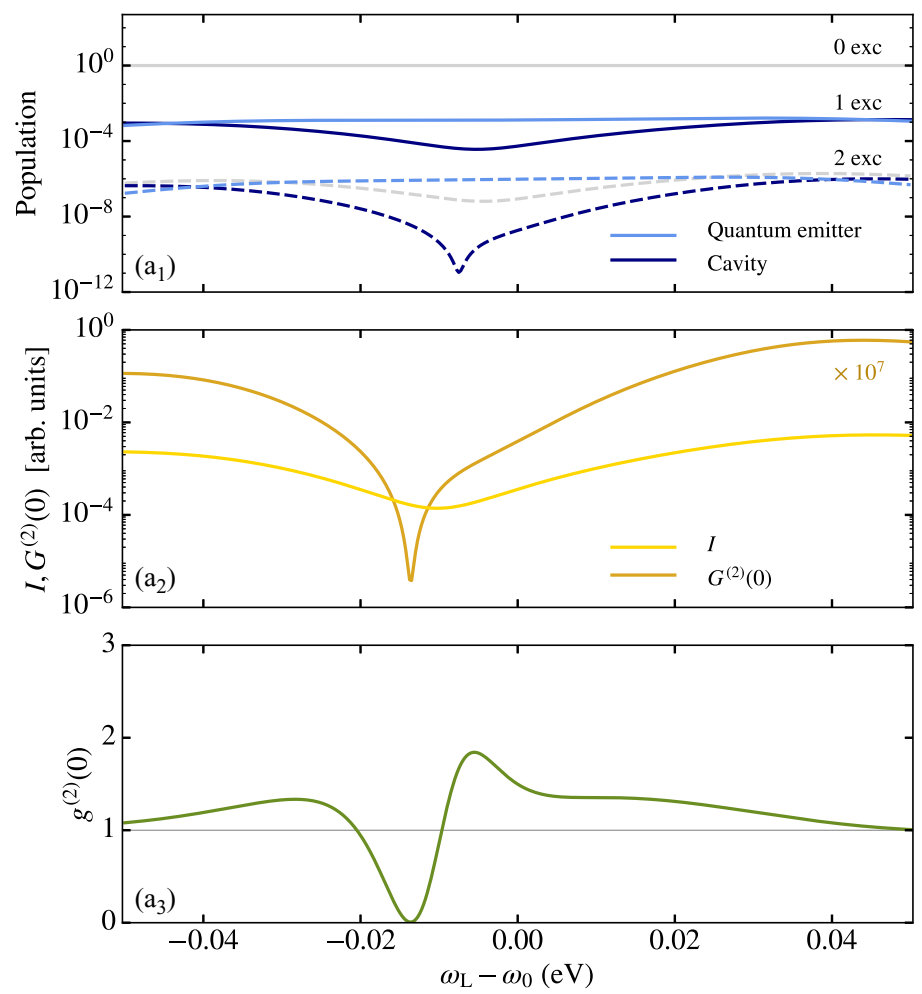

(b) Semiconductor microcavities
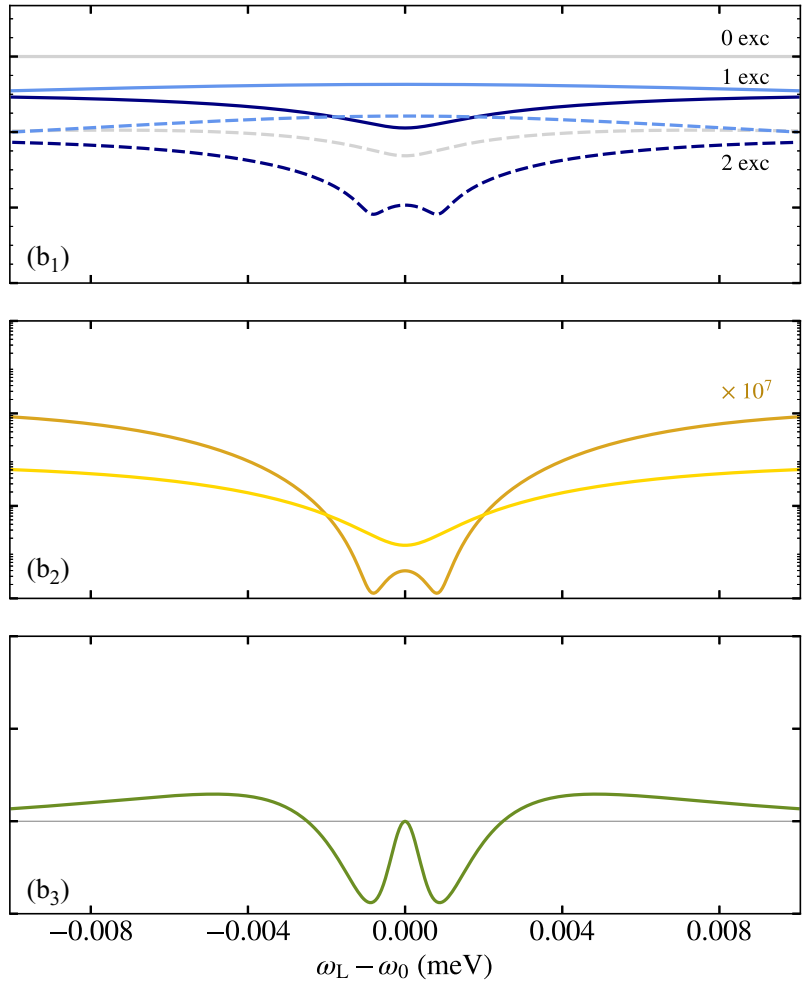

FIG. 6. Population (in the cavity-emitters basis), intensity $I$, and correlation functions $G^{(2)}(0)$ and $g^{(2)}(0)$ versus laser detuning $\omega_{\mathrm{L}}-\omega_{0}$ for a system of $N=5$ quantum emitters coupled to a nanocavity (a) and a microcavity (b) at coupling strengths $\lambda / \gamma_{\mathrm{C}}=0.2$ and 0.1 , respectively (for which the interference-induced correlations appear).

The intensity plots reflect the presence of the polariton energies as well: each scattering peak coincides with a maximum in the polariton population and, naturally, with the position of the polariton energy. The intensity minima are certainly located between the two polariton energies, far from resonance. The fact that the maxima in $G^{(2)}(0)$ are shifted from those in the scattered intensity provokes the characteristic shape in the normalized second-order correlation function, shown in Figs. $5\left(\mathrm{a}_{4}\right)$ and $5\left(\mathrm{~b}_{4}\right)$. Values of the $g^{(2)}(0)$ function below one are located close to the polariton energies (vertical continuous gray lines), whereas there appear two relative maxima at laser frequencies that match energy differences between the one- and the two-excitation manifolds (vertical dashed gray lines). For the nanocavity, the different positions of $I$ and $G^{(2)}(0)$ minima in Fig. 5( $\left.\mathrm{a}_{3}\right)$ leads to maxima and a minima in $g^{(2)}(0)$ near resonance, although the emission is always super-Poissonian in this frequency window.

\section{Interference-induced correlations}

The decrease of $g^{(2)}(0)$ below one is referred to as interference-induced correlations when its origin cannot be explained in terms of the energy levels as done for the photon blockade effect. On the contrary, it is produced by the destructive interference between different available decay paths [80-82].
The population curves [Figs. $6\left(a_{1}\right)$ and $6\left(b_{1}\right)$ ] reveal that it is a decrease in the population of the state corresponding to two cavity-mode excitations (dashed dark blue lines) that produces the minimum in the $G^{(2)}(0)$ function. It is then transferred to the normalized $g^{(2)}(0)$ and, consequently, there appears sub-Poissonian statistics in the vicinity of this laser frequency. This correspondence between cavity population and correlations is observed in both types of cavities, although there exists a difference between them: whereas only one dip takes place in nanocavities, two of them emerge in the case of microcavities. Notice that this behavior differs from the photon blockade mechanism, where a related fall in the population of the state with two cavity-mode excitations is not observed (on the contrary, as previously pointed out, all populations seem to converge to the same value).

The intensity $I$ and the correlation function $G^{(2)}(0)$ are depicted in Figs. $6\left(\mathrm{a}_{2}\right)$ and $6\left(\mathrm{~b}_{2}\right)$. As in the previous case, these curves clearly follow the shape of the populations associated with the states corresponding to one (continuous dark blue lines) and two (dashed dark blue lines) excitations in the cavity mode, respectively. The different position of the minima for these two magnitudes is again responsible for the shape of the $g^{(2)}(0)$ function. Nevertheless, now values below one are reached (the interference-induced photon correlations). In Fig. 5, the minimum in the $G^{(2)}$ did not lead to sub-Poissonian statistics, although it did correspond to a minimum in $g^{(2)}(0)$. Note that this fall was not so abrupt when compared with the intensity dip. 
(a) $\Theta=1 \gamma_{\mathrm{C}}$
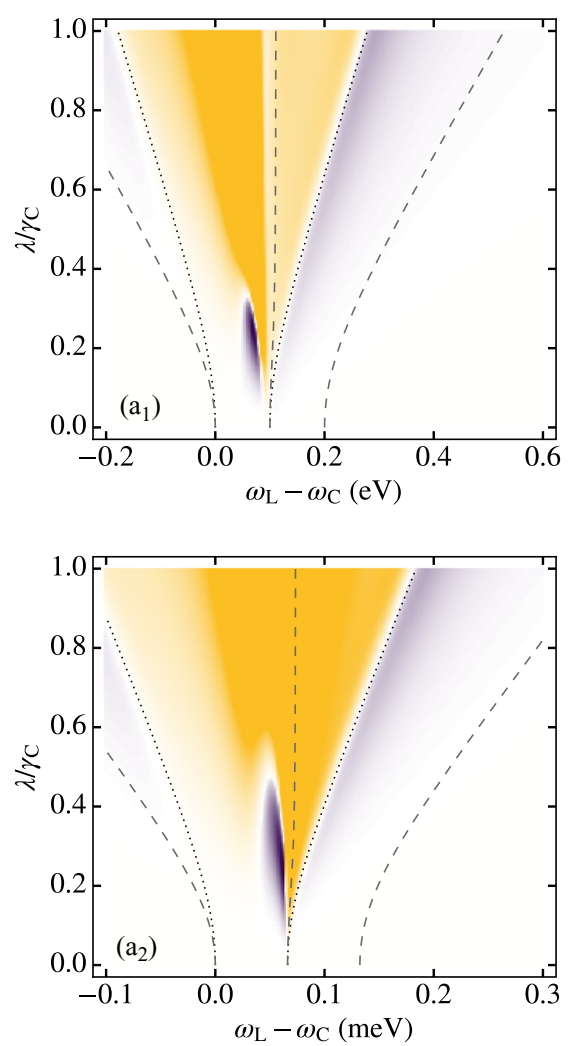

(b) $\Theta=2 \gamma_{\mathrm{C}}$
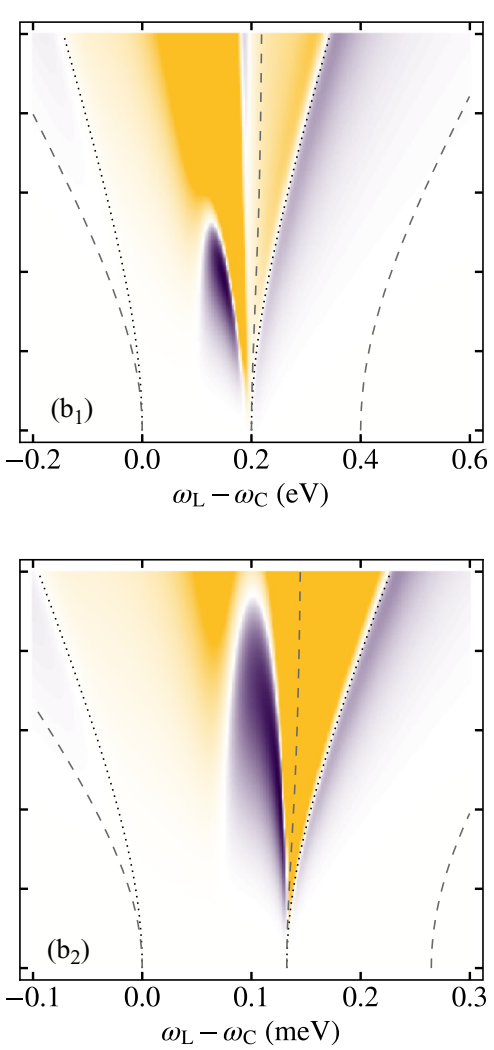

(c) $\Theta=3 \gamma_{C}$
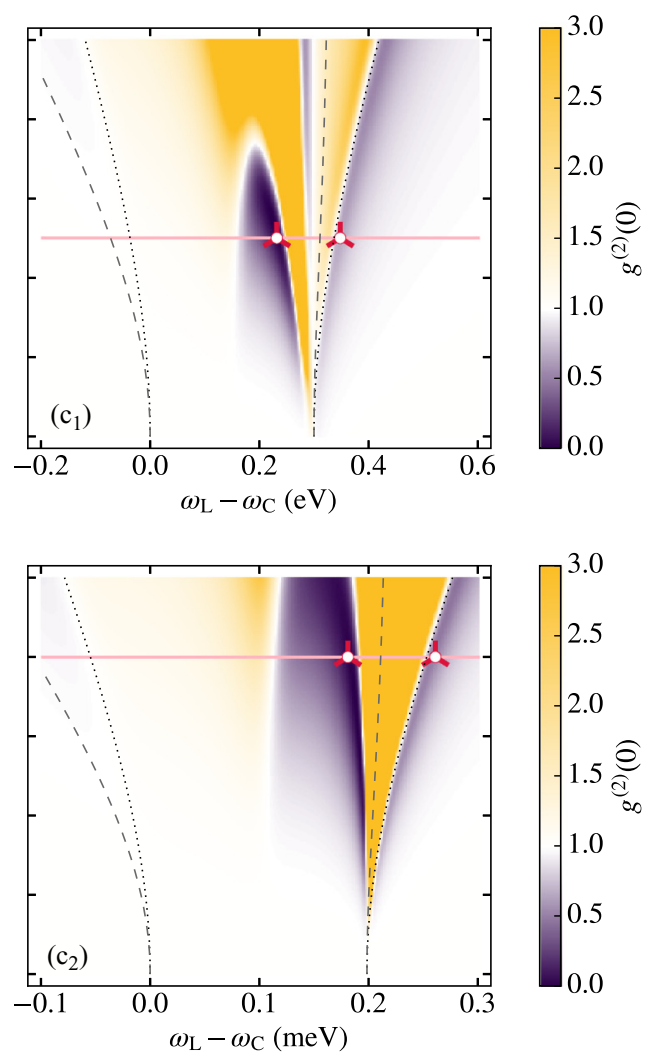

FIG. 7. Correlation function $g^{(2)}(0)$ versus laser detuning $\omega_{\mathrm{L}}-\omega_{\mathrm{C}}$ and coupling strength $\lambda$ (in units of the cavity decay rate $\gamma_{\mathrm{C}}$ ) for a system of $N=5$ quantum emitters coupled to a nanocavity (top row) and a microcavity (bottom row) for various values of the detuning between cavity and emitters, $\Theta=1 \gamma_{\mathrm{C}}(\mathrm{a}), 2 \gamma_{\mathrm{C}}(\mathrm{b})$, and $3 \gamma_{\mathrm{C}}$ (c). In these panels, dotted (dashed) lines plot the polariton frequencies (half-frequencies) in the one-excitation (two-excitation) manifold. Horizontal pink lines and magenta marks in (c) panels indicate points whose $g^{(2)}(0)$ is plotted in Fig. 9.

\section{Effect of detuning between cavity and emitter frequencies on the correlation function $g^{(2)}(0)$}

By means of the introduction of detuning between cavity and emitter frequencies, the parameter range in which subPoissonian statistics emerges can be enlarged: the spectral window becomes wider, and stronger couplings are required [54]. This is the tendency we observe in Fig. 7, where the correlation function $g^{(2)}(0)$ is plotted versus the laser detuning $\omega_{\mathrm{L}}-\omega_{\mathrm{C}}$ and the coupling strength $\lambda$ for various values of the detuning $\Theta \equiv \omega_{\mathrm{QE}}-\omega_{\mathrm{C}}$. There, the emitter frequencies $\omega_{\mathrm{QE}}$ vary while the cavity mode resonance is always fixed to be $\omega_{\mathrm{C}}=\omega_{0} \equiv 3 \mathrm{eV}$. The case considered is that composed of $N=5$ quantum emitters coupled to either a nanocavity (top row) or a microcavity (bottom row), hence, these would correspond to Figs. $2\left(b_{2}\right)$ and $4\left(b_{2}\right)$, respectively, if no detuning were present (that is, $\Theta=0$ ).

Figure 7 shows that, effectively, for both types of cavities the region with interference-induced correlations spreads as the difference in energy between cavity and emitters increases, although this effect is more pronounced in microcavities. Via detuning, the range of laser frequencies for which subPoissonian emission is attainable broadens: it extends over a frequency window with a width of almost half the detuning.
Focusing now on the vertical axis, we observe that for a particular value of the coupling strength, it is possible to have $g^{(2)}(0)<1$ near the resonant frequency just by increasing the detuning between cavity and emitters. Furthermore, note that the introduction of detuning makes it possible to achieve lower values of $g^{(2)}(0)$, whereby improving the quantum character of the emitted light. This is also true for the photon blockade effect following the UP (since this is the dressed state with a greater emitter contribution in this case), which deepens. For a better visualization, the energies corresponding to the eigenvalues of the dressed states are plotted in dotted (one-excitation manifold) and dashed (two-excitation manifold) lines in all panels. For both nanocavities and microcavities, the photon blockade effect reinforces near the UP, whereas it fades at the LP. For instance, when $\Theta=3 \gamma_{\text {C }}$ the photon blockade effect following the lower branch disappears; no sub-Poissonian emission takes place in its surroundings. Note that varying the sign of the detuning, the roles of UP and LP are exchanged.

Regarding the region with interference-induced correlations, there exists a particularity for the microcavity that is worth mentioning: now we only observe one prevailing dip, instead of two (as it was for the zero detuning case, $\Theta=0$ ). As a consequence of the loss of symmetry, the dip closer to 
(a) Plasmonic nanocavities
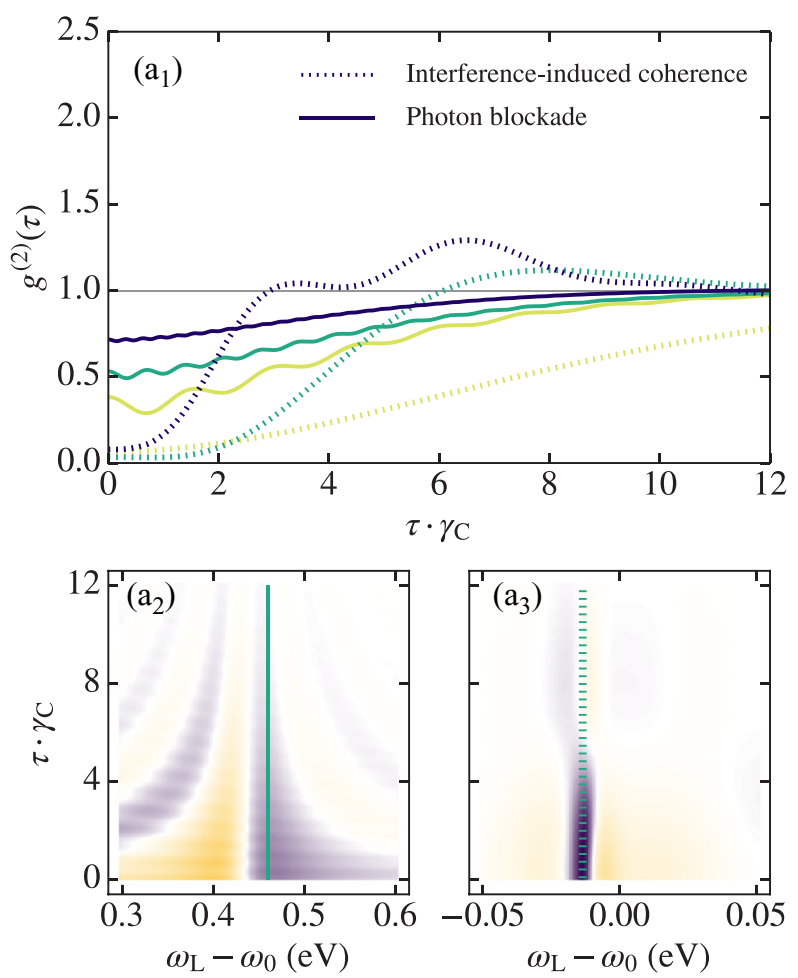

(b) Semiconductor microcavities
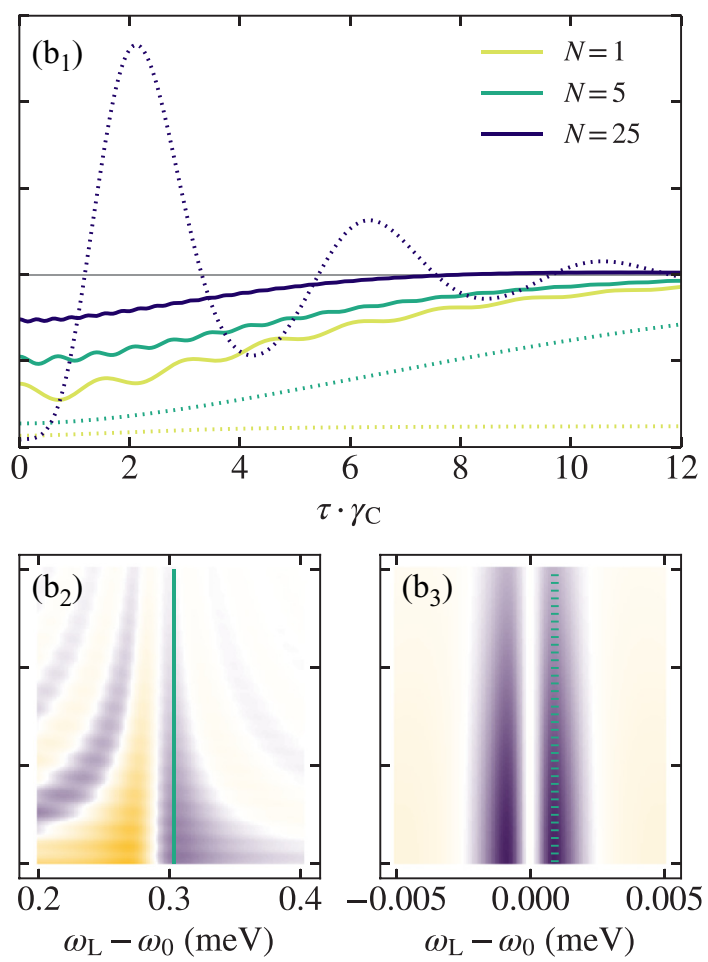

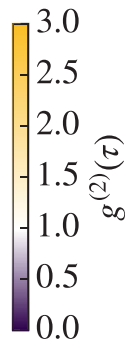

.0

FIG. 8. Correlation function $g^{(2)}(\tau)$ for $N$ quantum emitters interacting with either a nanocavity (a) or a microcavity (b) at resonance $(\Theta=0)$. In top panels, $g^{(2)}(\tau)$ is plotted versus the time delay $\tau$ (in units of the cavity time $\left.1 / \gamma_{\mathrm{C}}\right)$ for three different ensemble sizes $(N=1$, 5, and 25). Two different configurations (which correspond to the points indicated in Figs. 2 and 4) are selected. Continuous lines are used for the antibunching related to the photon blockade effect while dotted lines are used for interference-induced correlations. In bottom panels, $g^{(2)}(\tau)$ is plotted versus laser detuning $\omega_{\mathrm{L}}-\omega_{0}$ and time delay $\tau$ (also in units of $1 / \gamma_{\mathrm{C}}$ ) for an ensemble of $N=5$ emitters and selecting two different coupling strengths in each case: $\lambda=2 \gamma_{\mathrm{C}}\left(\mathrm{a}_{2}\right)$ and $0.2 \gamma_{\mathrm{C}}\left(\mathrm{a}_{3}\right)$ for plasmonic nanocavities and $\lambda=2 \gamma_{\mathrm{C}}\left(\mathrm{b}_{2}\right)$ and $0.1 \gamma_{\mathrm{C}}\left(\mathrm{b}_{3}\right)$ for dielectric microcavities. In these bottom panels, vertical green lines correspond to the curves for $N=5$ depicted at the top.

the emitter frequency becomes narrower, and the other one widens, when the detuning increases. This makes the patterns observed for $g^{(2)}(0)$ at a specific detuning $\Theta$ quite similar for nanocavities and microcavities. Nevertheless, the energy range is very different; note that the coupling strength $\lambda$ is given in units of the decay rate $\gamma_{\mathrm{C}}$, and the values corresponding to plasmonic $\left(\gamma_{\mathrm{C}} \sim 0.1 \mathrm{eV}\right)$ and dielectric $\left(\gamma_{\mathrm{C}} \sim 0.1 \mathrm{meV}\right)$ cavities differ by around three orders of magnitude (as do the laser detunings).

\section{Dependence of the correlation function $g^{(2)}(\tau)$ on the time delay $\tau$}

In this section, we study the behavior of the secondorder correlation function $g^{(2)}(\tau)$ at nonzero time delays $\tau$ for various configurations displaying sub-Poissonian statistics $\left[g^{(2)}(0)<1\right]$. This allows to resolve whether the emitted light is actually antibunched $\left[g^{(2)}(0)<g^{(2)}(\tau)\right]$. In top panels of Fig. 8, we plot $g^{(2)}(\tau)$ as a function of $\tau$ (in units of the cavity lifetime $1 / \gamma_{\mathrm{C}}$ ) for an ensemble of $N$ quantum emitters interacting with either a nanocavity (a) or a microcavity (b) when there is no detuning between them $(\Theta=0)$. We consider three ensemble sizes $N=1$ (yellow lines), 5 (green lines), and 25 (blue lines), and select two different configurations for each case: one belonging to the photon blockade area (continuous lines) and the another displaying sub-Poissonian statistics due to quantum interference effects (dotted lines). All configurations are indicated in Figs. 2 and 4 through magenta marks.

Focusing first on the continuous lines (photon blockade), we observe that the correlation function approaches one almost monotonically as the time interval $\tau$ increases, hence, we can talk properly of photon antibunching. Nevertheless, there exist some oscillations whose amplitude diminishes as the ensemble size increases. Remarkably, there is practically no difference between the behavior for nanocavities and microcavities once the timescale is normalized by the cavity decay time $1 / \gamma_{C}$; in both cases, the time evolution follows the same tendency, and the degree of correlation reached from both effects is similar. Note that all configurations have been chosen for a coupling strength $\lambda / \gamma_{C}=2$ for both types of cavities, although the laser detuning varies in order to consider the minimum of the $g^{(2)}(0)$ attainable at this coupling. These two sets of curves also highlight that the degree of coherence is quickly lost when increasing $N$.

Dotted lines show instead the evolution in $\tau$ for configurations displaying interference-induced correlations. We first observe that the degree of coherence at $\tau=0$ reached from this effect is stronger than the associated with the photon blockade mechanism, although the couplings are smaller: for 
(a) Plasmonic nanocavities
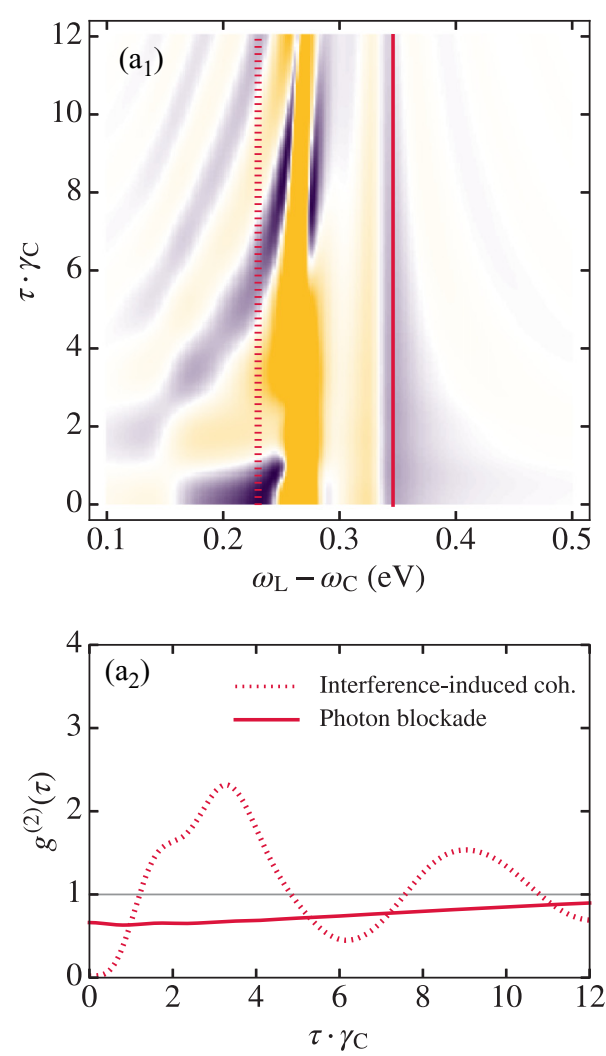

(b) Semiconductor microcavities
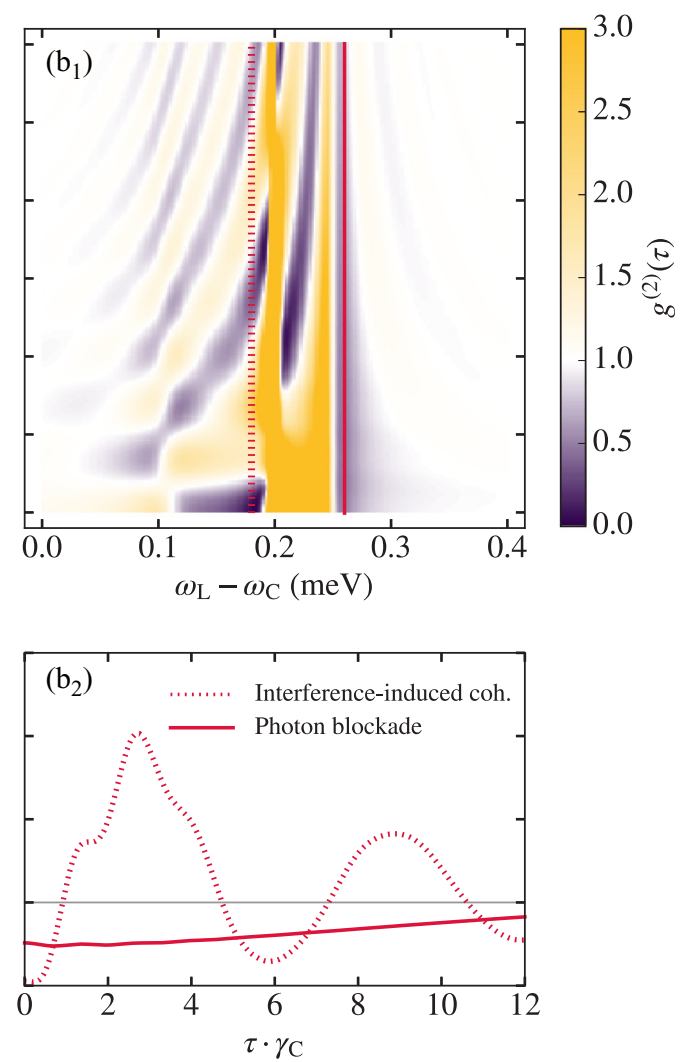

FIG. 9. Correlation function $g^{(2)}(\tau)$ for an ensemble of $N=5$ quantum emitters interacting with either a nanocavity (a) or a microcavity (b) cavity when $\Theta=3 \gamma_{\mathrm{C}}$. In top panels, $g^{(2)}(\tau)$ is plotted versus laser detuning $\omega_{\mathrm{L}}-\omega_{\mathrm{C}}$ and time interval $\tau$ (in units of the cavity time $1 / \gamma_{\mathrm{C}}$ ) for coupling strength $\lambda=0.5 \gamma_{\mathrm{C}}$ in (a) and $\lambda=0.8 \gamma_{\mathrm{C}}$ in (b). The cuts at $\tau=0$ correspond to the continuous pink lines depicted in Fig. 7(c). Bottom panels show cuts of the contour plots on top at laser detunings yielding two minima in the $g^{(2)}(0)$ function. These specific configurations are indicated by red lines in the top panels and by red markers in 7(c).

the nanocavity, $\lambda / \gamma_{\mathrm{C}}=0.2$, while for the microcavity it takes the values $\lambda / \gamma_{C}=0.04,0.1$, and 0.3 for $N=1,5$, and 25 , respectively. Oscillations in the $\tau$ evolution of the function $g^{(2)}(\tau)$ are observed as $N$ increases, although we still have antibunched light since $g^{(2)}(0)<g^{(2)}(\tau)$. When comparing nanocavities and microcavities, we observe that oscillations in the latter are more pronounced. Moreover, note again the difference in $\gamma_{\mathrm{C}}$, which translates into the fact that the temporal evolution is significantly faster in the plasmonic nanocavity (a direct consequence of the spectrally broad character of photon correlations in the system).

A more general picture is shown in the bottom row of Fig. 8, where $g^{(2)}(\tau)$ is plotted as a function of the laser detuning $\omega_{\mathrm{L}}-\omega_{0}$ and the time delay $\tau$ (again in units of the cavity time $1 / \gamma_{\mathrm{C}}$ ) for a collection of $N=5$ emitters also interacting with either a nanocavity (a) or a microcavity (b) for specific coupling strengths (see Fig. 8 caption). The particular values of the laser detuning marked with vertical green lines (continuous for photon blockade and dotted for interference-induced correlations) correspond to the ones depicted in Figs. 8( $\left.a_{1}\right)$ and 8( $\left.b_{1}\right)$ for $N=5$. These contour plots show that oscillatory patterns are also present for configurations displaying super-Poissonian statistics at zero-time delay.

We have thus found that sub-Poissonian statistics is accompanied by antibunched light in the zero-detuning configura- tions explored in Fig. 8. Although $g^{(2)}(\tau)$ approaches one as the time delay increases, its evolution is far from monotonous for interference-induced correlations: they present an oscillatory pattern taking values above and below one before reaching the coherent limit. This also happens when detuning between the cavity frequency and the emitters is introduced. An example is shown in the top row of Fig. 9, where the function $g^{(2)}(\tau)$ is plotted for a particular coupling strength as a function of laser detuning $\omega_{\mathrm{L}}-\omega_{\mathrm{C}}$, and the time delay $\tau$ (in units of $1 / \gamma_{\mathrm{C}}$ ) for $N=5$ quantum emitters interacting with either a nanocavity (a) or a microcavity (b). Here, we have considered a detuning $\Theta=3 \gamma_{\mathrm{C}}$, so these plots correspond to horizontal cuts in the panels of the third column of Fig. 7 (indicated by horizontal pink lines), at $\lambda / \gamma_{\mathrm{C}}=0.5$ for plasmonic nanocavities and $\lambda / \gamma_{\mathrm{C}}=0.8$ for dielectric microcavities. In these panels, for most laser detunings, the correlation function develops an oscillatory pattern as $\tau$ increases for both sub- and superPoissonian statistics at $\tau=0$. Again, the close similarity between the patterns for both cavities is remarkable (once the delay time is expressed in units of $1 / \gamma_{\mathrm{C}}$ ).

We observe that there exists a significant difference in the temporal dependence of negative correlations also once detuning between cavity and emitters is introduced. This is evident in the bottom panels of Fig. 9, where two specific values of $\omega_{\mathrm{L}}-\omega_{\mathrm{C}}$ are considered (indicated in Fig. 7 with 
magenta marks) in order to select configurations that display sub-Poissonian statistics due to interference effects (dotted line) and photon blockade (continuous line). These plots of $g^{(2)}(\tau)$ versus the time delay correspond to vertical cuts in Figs. $9\left(a_{1}\right)$ and $9\left(b_{1}\right)$ (see vertical red lines). In the case of photon blockade, $g^{(2)}(\tau)$ approaches one monotonically as the delay increases. In contrast, quantum interference leads to an oscillatory pattern in correlations. Both retain a temporal evolution similar to the one obtained at $\Theta=0$ in Fig. 8 . Note that even the temporal slope and pitch of oscillations remain the same. Thus, by detuning cavity and emitters, the opportunity to obtain sub-Poissonian light improves (as we have mentioned before, the parameter regions widen) without altering qualitatively its evolution with time delay between photon detections. Again, the phenomenologies for nanocavities and microcavities coincide, given that the values of laser detuning and time delay, both normalized to the cavity losses, are the same.

\section{CONCLUSIONS}

This work investigates the statistical properties of the light generated by a collection of quantum emitters coupled to a single electromagnetic mode. Theoretical computations based on an effective Hamiltonian approach have been carried out to describe the response of two different systems under lowintensity coherent driving: plasmonic nanocavities and dielectric microcavities. Special attention has focused on exploring the impact that the distinct open or closed character of these two types of cavities has on the scattered light.

For both cavity configurations, sub-Poissonian emission has been observed not only at the single-emitter level, but also for mesoscopic ensembles involving several tens of emitters. Our results show that there are two different mechanisms that yield significant negative correlations in the interaction between a purely bosonic subsystem (cavity) and a quasibosonic one (emitter ensemble): photon blockade and destructive interference. The former takes place at high coupling strengths (comparable to or larger than the cavity decay rate), while the latter becomes relevant for weaker cavity-emitters interactions. Despite their distinct open or closed character and the largely different physical parameters describing nanocavities and microcavities, the photon statistics phenomenology for both systems is remarkably similar (once normalized to the cavity losses). This fact becomes clearer through the exploration of cavity-emitters spectral detuning, which enlarges the parameter range yielding antibunched light, and the temporal evolution of correlations, which reveals the slow (fast) fading of photon blockade (interference-induced) antibunching. Our findings may serve as guidance for the optimization of quantum optical phenomena for specific applications through the appropriate choice of material parameters for their implementation.

\section{ACKNOWLEDGMENT}

This work has been funded by the European Research Council under Grant Agreements No. ERC-2011-AdG 290981 and No. ERC-2016-STG-714870, the EU Seventh Framework Programme (Grants No. FP7-PEOPLE-2013-CIG-630996 and No. FP7-PEOPLE-2013-CIG-618229), and the Spanish MINECO under Contracts No. MAT2014-53432-C5-5-R and No. FIS2015-64951-R, as well as through the "María de Maeztu" programme for Units of Excellence in R\&D (Grant No. MDM-2014-0377).
[1] A. V. Kavokin, J. J. Baumberg, G. Malpuech, and F. P. Laussy, Microcavities (Oxford University Press, New York, 2007).

[2] E. T. Jaynes and F. W. Cummings, Proc. IEEE 51, 89 (1963).

[3] M. Tavis and F. W. Cummings, Phys. Rev. 170, 379 (1968).

[4] H.-P. Breuer and F. Petruccione, The Theory of Open Quantum Systems (Oxford University Press, Oxford, 2002).

[5] P. Michler, A. Kiraz, L. Zhang, C. Becher, E. Hu, and A. Imamoğlu, Appl. Phys. Lett. 77, 184 (2000).

[6] S. Reitzenstein, A. Bazhenov, A. Gorbunov, C. Hofmann, S. Münch, A. Löffler, M. Kamp, J. P. Reithmaier, V. D. Kulakovskii, and A. Forchel, Appl. Phys. Lett. 89, 051107 (2006).

[7] M. Gross and S. Haroche, Phys. Rep. 93, 301 (1982).

[8] M. Scheibner, T. Schmidt, L. Worschech, A. Forchel, G. Bacher, T. Passow, and D. Hommel, Nat. Phys. 3, 106 (2007).

[9] A. Faraon, I. Fushman, D. Englund, N. Stoltz, P. Petroff, and J. Vučković, Nat. Phys. 4, 859 (2008).

[10] J. M. Raimond, M. Brune, and S. Haroche, Rev. Mod. Phys. 73, 565 (2001).

[11] T. Pellizzari, S. A. Gardiner, J. I. Cirac, and P. Zoller, Phys. Rev. Lett. 75, 3788 (1995).

[12] A. Imamoğlu, D. D. Awschalom, G. Burkard, D. P. DiVincenzo, D. Loss, M. Sherwin, and A. Small, Phys. Rev. Lett. 83, 4204 (1999).

[13] K. J. Vahala, Nature (London) 424, 839 (2003).
[14] J. M. Gérard, D. Barrier, J. Y. Marzin, R. Kuszelewicz, L. Manin, E. Costard, V. Thierry-Mieg, and T. Rivera, Appl. Phys. Lett. 69, 449 (1996).

[15] A. J. Bennett, D. C. Unitt, P. See, A. J. Shields, P. Atkinson, K. Cooper, and D. A. Ritchie, Appl. Phys. Lett. 86, 181102 (2005).

[16] B. Gayral, J. M. Gérard, A. Lemaître, C. Dupuis, L. Manin, and J. L. Pelouard, Appl. Phys. Lett. 75, 1908 (1999).

[17] D. W. Vernooy, V. S. Ilchenko, H. Mabuchi, E. W. Streed, and H. J. Kimble, Opt. Lett. 23, 247 (1998).

[18] K. Srinivasan, P. E. Barclay, O. Painter, J. Chen, A. Y. Cho, and C. Gmachl, Appl. Phys. Lett. 83, 1915 (2003).

[19] J. Vučković and Y. Yoshihisa, Appl. Phys. Lett. 82, 2374 (2003).

[20] Y. Akahane, T. Asano, B.-S. Song, and S. Noda, Nature (London) 425, 944 (2003).

[21] E. M. Purcell, H. C. Torrey, and R. V. Pound, Phys. Rev. 69, 37 (1946).

[22] C. Weisbuch, M. Nishioka, A. Ishikawa, and Y. Arakawa, Phys. Rev. Lett. 69, 3314 (1992).

[23] R. Houdre, R. P. Stanley, U. Oesterle, M. Ilegems, and C. Weisbuch, Phys. Rev. B 49, 16761 (1994).

[24] J. Bellessa, C. Bonnand, J. C. Plenet, and J. Mugnier, Phys. Rev. Lett. 93, 036404 (2004).

[25] T. Schwartz, J. A. Hutchison, C. Genet, and T. W. Ebbesen, Phys. Rev. Lett. 106, 196405 (2011). 
[26] J. McKeever, A. Boca, A. D. Boozer, J. R. Buck, and H. J. Kimble, Nature (London) 425, 268 (2003).

[27] J. P. Reithmaier, A. L. G. Sęk, C. Hofmann, S. Kuhn, S. Reitzenstein, L. V. Keldysh, V. D. Kulakovskii, T. L. Reinecke, and A. Forchel, Nature (London) 432, 197 (2004).

[28] T. Yoshie, A. Scherer, J. Hendrickson, G. Khitrova, H. M. Gibbs, G. Rupper, C. Ell, O. B. Shchekin, and D. G. Deppe, Nature (London) 432, 200 (2004).

[29] R. Chikkaraddy, B. D. Nijs, F. Benz, S. J. Barrow, O. A. Scherman, E. Rosta, A. Demetriadou, P. Fox, O. Hess, and J. Baumberg, Nature (London) 535, 127 (2016).

[30] K. Santhosh, O. Bitton, L. Chuntonov, and G. Haran, Nat. Commun. 7, 11823 (2016).

[31] R. H. Dicke, Phys. Rev. 93, 99 (1954).

[32] A. Delga, J. Feist, J. Bravo-Abad, and F. J. García-Vidal, Phys. Rev. Lett. 112, 253601 (2014).

[33] A. González-Tudela, P. A. Huidobro, L. Martín-Moreno, C. Tejedor, and F. J. García-Vidal, Phys. Rev. Lett. 110, 126801 (2013).

[34] N. Shammah, N. Lambert, F. Nori, and S. DeLiberato, Phys. Rev. A 96, 023863 (2017).

[35] F. Alpeggiani, S. D'Agostino, D. Sanvitto, and D. Gerace, Sci. Rep. 6, 34772 (2016).

[36] R. Sáez-Blázquez, J. Feist, A. I. Fernández-Domínguez, and F. J. García-Vidal, Optica 4, 1363 (2017).

[37] H. J. Kimble, M. Dagenais, and L. Mandel, Phys. Rev. Lett. 39, 691 (1977).

[38] J. C. López Carreño, E. Zubizarreta Casalengua, E. del Valle, and F. P. Laussy, arXiv:1610.06126.

[39] C. H. Bennett, G. Brassard, and A. K. Ekert, Sci. Am. 267, 50 (1992).

[40] C. L. Degen, F. Reinhard, and P. Cappellaro, Rev. Mod. Phys. 89, 035002 (2017).

[41] V. Giovannetti, S. Lloyd, and L. Maccone, Phys. Rev. Lett. 96, 010401 (2006).

[42] H. J. Kimble, Nature (London) 453, 1023 (2008).

[43] B. Lounis and M. Orrit, Rep. Prog. Phys. 68, 1129 (2005).

[44] A. Muller, T. Herzog, B. Huttner, W. Tittel, H. Zbinden, and N. Gisin, Appl. Phys. Lett. 70, 793 (1997).

[45] A. Kuhn, M. Hennrich, and G. Rempe, Phys. Rev. Lett. 89, 067901 (2002).

[46] B. Lounis and W. E. Moerner, Nature 407, 491 (2000).

[47] I. Aharonovich, S. Castelletto, D. A. Simpson, C.-H. Su, A. D. Greentree, and S. Prawer, Rep. Prog. Phys. 74, 076501 (2011).

[48] X. Brokmann, E. Giacobino, M. Dahana, and J. P. Hermier, Appl. Phys. Lett. 85, 712 (2004).

[49] C. K. Hong and L. Mandel, Phys. Rev. Lett. 56, 58 (1986).

[50] G. Rempe, R. J. Thompson, R. J. Brecha, W. D. Lee, and H. J. Kimble, Phys. Rev. Lett. 67, 1727 (1991).

[51] H. J. Carmichael, R. J. Brecha, and P. R. Rice, Opt. Commun. 82, 73 (1991).

[52] G. T. Foster, S. L. Mielke, and L. A. Orozco, Phys. Rev. A 61, 053821 (2000).

[53] A. Aufféves, D. Gerace, S. Portolan, A. Drezet, and M. F. Santos, New J. Phys. 13, 093020 (2011).
[54] M. Radulaski, K. A. Fischer, K. G. Lagoudakis, J. L. Zhang, and J. Vučković, Phys. Rev. A 96, 011801(R) (2017).

[55] D. Meiser and M. J. Holland, Phys. Rev. A 81, 063827 (2010).

[56] M. Richter, M. Gegg, T. S. Theuerholz, and A. Knorr, Phys. Rev. B 91, 035306 (2015).

[57] A. Grankin, E. Brion, E. Bimbard, R. Boddeda, I. Usmani, A. Ourjoumtsev, and P. Grangier, New J. Phys. 16, 043020 (2014).

[58] V. V. Temnov and U. Woggon, Phys. Rev. Lett. 95, 243602 (2005).

[59] V. V. Temnov and U. Woggon, Opt. Express 17, 5774 (2009).

[60] M. Gegg and M. Richter, New J. Phys. 18, 043037 (2016).

[61] S. Savasta, R. Saija, A. Ridolfo, O. D. Stefano, P. Denti, and F. Borghese, ACS Nano 4, 6369 (2010).

[62] X.-W. Chen, V. Sandoghdar, and M. Agio, Phys. Rev. Lett. 110, 153605 (2013).

[63] G. Zengin, M. Wersäll, S. Nilsson, T. J. Antosiewicz, M. Käll, and T. Shegai, Phys. Rev. Lett. 114, 157401 (2015).

[64] F. Peyskens, D. Chang, and D. Englund, Phys. Rev. B 96, 235151 (2017).

[65] M. S. Tame, K. R. McEnery, S. K. Özdemir, J. Lee, S. A. Maier, and M. S. Kim, Nat. Phys. 9, 329 (2013).

[66] D. Englund, A. Faraon, I. Fushman, N. Stoltz, P. Petroff, and J. Vučković, Nature (London) 450, 857 (2007).

[67] J. L. Jewell, S. L. McCall, Y. H. Lee, A. Scherer, A. C. Gossard, and J. H. English, Appl. Phys. Lett. 54, 1400 (1989).

[68] D. F. Walls and G. J. Milburn, Quantum Optics, 2nd ed. (Springer, Berlin, 2008), p. 199.

[69] R. Loudon, The Quantum Theory of Light (Oxford University Press, Oxford, 1973).

[70] X. T. Zou and L. Mandel, Phys. Rev. A 41, 475 (1990).

[71] D. F. Walls and G. J. Milburn, Quantum Optics, 2nd ed. (Springer, Berlin, 2008), pp. 38-42.

[72] L. Novotny and B. Hecht, Principles of Nano-optics (Cambridge University Press, Cambridge, England, 2006), p 276.

[73] S. A. Maier, Plasmonics: Fundamentals and Applications (Springer, New York, 2007), pp. 73-77.

[74] C. González-Ballestero, J. Feist, E. Moreno, and F. J. GarcíaVidal, Phys. Rev. B 92, 121402(R) (2015).

[75] A. Thoma, P. Schnauber, M. Gschrey, M. Seifried, J. Wolters, J.-H. Schulze, A. Strittmatter, S. Rodt, A. Carmele, A. Knorr, T. Heindel, and S. Reitzenstein, Phys. Rev. Lett. 116, 033601 (2016).

[76] K. D. Rector and M. D. Faye, J. Chem. Phys. 108, 1794 (1998).

[77] W. Zhang, A. O. Govorov, and G. W. Bryant, Phys. Rev. Lett. 97, 146804 (2006).

[78] R. D. Artuso and G. W. Bryant, Nano Lett. 8, 2106 (2008).

[79] A. Ridolfo, O. Di Stefano, N. Fina, R. Saija, and S. Savasta, Phys. Rev. Lett. 105, 263601 (2010).

[80] T. C. H. Liew and V. Savona, Phys. Rev. Lett. 104, 183601 (2010).

[81] M. Bamba, A. Imamoğlu, I. Carusotto, and C. Ciuti, Phys. Rev. A 83, 021802(R) (2011).

[82] I. Carusotto and C. Ciuti, Rev. Mod. Phys. 85, 299 (2013). 